Article

\title{
Evaluation of Investment Policy Based on Multi-Attribute Decision-Making Using Interval Valued T-Spherical Fuzzy Aggregation Operators
}

\author{
Kifayat Ullah $^{1}(\mathbb{D})$, Nasruddin Hassan ${ }^{2, *(\mathbb{D})}$, Tahir Mahmood ${ }^{1}\left(\mathbb{D}\right.$, Naeem $\operatorname{Jan}^{1}$ and \\ Mazlan Hassan ${ }^{3}$ (D) \\ 1 Department of Mathematics and Statistics, International Islamic University, Islamabad 44000, Pakistan; \\ kifayat.phdma72@iiu.edu.pk (K.U.); tahirbakhat@iiu.edu.pk (T.M.); naeem.phdma73@iiu.edu.pk (N.J.) \\ 2 School of Mathematical Sciences, Faculty of Science and Technology, Universiti Kebangsaan Malaysia, \\ Bangi 43600, Selangor, Malaysia \\ 3 Department of Management and Marketing, Faculty of Economics and Management, \\ Universiti Putra Malaysia, Serdang 43400, Selangor, Malaysia; mazlanhs@upm.edu.my \\ * Correspondence: nas@ukm.edu.my; Tel.: +60-1-9214-5750
}

Received: 20 January 2019; Accepted: 4 March 2019; Published: 9 March 2019

\begin{abstract}
Expressing the measure of uncertainty, in terms of an interval instead of a crisp number, provides improved results in fuzzy mathematics. Several such concepts are established, including the interval-valued fuzzy set, the interval-valued intuitionistic fuzzy set, and the interval-valued picture fuzzy set. The goal of this article is to enhance the T-spherical fuzzy set (TSFS) by introducing the interval-valued TSFS (IVTSFS), which describes the uncertainty measure in terms of the membership, abstinence, non-membership, and the refusal degree. The novelty of the IVTSFS over the pre-existing fuzzy structures is analyzed. The basic operations are proposed for IVTSFSs and their properties are investigated. Two aggregation operators for IVTSFSs are developed, including weighted averaging and weighted geometric operators, and their validity is examined using the induction method. Several consequences of new operators, along with their comparative studies, are elaborated. A multi-attribute decision-making method in the context of IVTSFSs is developed, followed by a brief numerical example where the selection of the best policy, among a list of investment policies of a multinational company, is to be evaluated. The advantages of using the framework of IVTSFSs are described theoretically and numerically, hence showing the limitations of pre-existing aggregation operators.
\end{abstract}

Keywords: aggregation operators; interval-valued T-spherical fuzzy set; multi-attribute decisionmaking; T-spherical fuzzy set

\section{Introduction}

Quantifying the uncertainty or imprecision of an event by a crisp number on a scale of zero to one has always been a tough task. The story began with Zadeh's model of the fuzzy set (FS) [1], where the imprecision of an event is expressed by a crisp number from zero to one [0,1]. Soon after the idea of the FS was introduced, it was realized that it is indeed a tough job to describe the uncertainty using crisp numbers. Therefore, the concept of the interval-valued fuzzy set (IVFS) [2] was developed, where the membership grade is described in terms of a closed sub-interval of zero to one $[0,1]$ rather than by crisp numbers. Representing the imprecision using an interval instead of a crisp number seems more real.

In 1986, Atanassov developed the notion of the intuitionistic fuzzy set (IFS) [3], introducing the concept of the non-membership grade along with a membership grade to model a real-life 
phenomenon, which was then enhanced to interval-valued IFS (IVIFS) [4]. It basically bounds the sum of the membership and non-membership grades between zero and one. Pointing out the limitations of IFS, Yager [5] introduced the idea of the Pythagorean fuzzy set (PyFS), which is also based on two characteristic functions but with a larger domain, by bounding the sum of the squares of the membership and non-membership grades between zero and one. Nevertheless, the PyFS failed to justify the situation when the sum of two grades crosses the boundary of zero to one. To describe such situations, Yager [6] introduced the notion of the q-rung ortho pair fuzzy sets (q-ROPFSs), which generalize both IFS as well as PyFS by enlarging their domains. A q-ROPFS allows the sum of the q-th power of the membership and non-membership grades to be between zero and one. The fuzzy structures of the interval-valued PyFS (IVPyFS), as well as the interval-valued q-ROPFS (IVq-ROPFS), have also been introduced by Peng and Yang [7] and Joshi et al. [8] due to the importance of expressing fuzziness in terms of intervals instead of crisp numbers. Further research work on IFSs, PyFSs, IVIFSs, and IVPyFSs is extensive [9-16].

Cuong [17] introduced a novel concept of the picture fuzzy set (PFS) by pointing out a special situation where one needed four characteristic functions to model a real-life event, such as voting. Cuong's model of the PFS created a new dimension in the field of fuzzy algebraic structures and scientists started to contribute to the development of PFSs due to its significance [18-21]. Cuong's PFS possessed the same problem as the IFS had, i.e., it bound the sum of the membership, abstinence, and non-membership grades between zero and one. This restriction does not allow the decision makers to select the values of three characteristic functions by their own choice. Realizing the issue, Mahmood et al. [22] developed a new model of the spherical fuzzy set (SFS) and the T-spherical fuzzy set (TSFS). A TSFS binds the sum of the q-th power of the membership, abstinence, and non-membership between zero and one and, hence, was declared as a diverse structure compared to the PFS, PyFS, and IFS. Due to the larger variety of characteristic functions with no limitation, several much-needed ideas can be developed in the framework of TSFSs, such as the aggregation theory of TSFSs, graphs of TSFSs, and similarity and distance measures of TSFSs. Some work on the aggregation of TSFSs, relations of TSFSs, and similarity measures of TSFSs is abound [22,23].

MADM (multi-attribute decision-making) models, based on weighted averaging (WA) and weighted geometric (WG) operators, are hot research topics in the field of fuzzy algebraic structures. The theory of the WA and WG aggregation operators of IFSs has been developed by $\mathrm{Xu}$ [24] and $\mathrm{Xu}$ and Yager [25], before being further applied in MADM problems. Some generalized WA, induced generalized WA, and induced generalized WG aggregation operators for IFSs have also been developed [26-28] and their applications in MADM problems have been demonstrated. The WA and WG operators for IVIFSs are proposed by Wang [29] and Wei and Wang [30], respectively, while some generalized WA and generalized WG operators for IVIFSs have been developed by Yu [31] and $\mathrm{Xu}$ and Cai [32], respectively. Some other useful tools in the aggregation of IFSs and IVIFSs, along with their applications, were also constructed [33-38]. For PyFSs, Rahman et al. [39] and Peng and Yang [40] developed the Pythagorean fuzzy WA and WG aggregation operators. The WA and WG aggregation tools for IVPyFSs [41,42] were formulated and the recent advancement on the theory of the aggregation of PyFSs and IVPyFSs, alongside their applications in MADM, are discussed [43-47]. The MADM and aggregation theory of hesitant fuzzy sets (HFSs) and bipolar-valued HFSs have also been developed [48-50]. Due to their enhanced and diverse structure, PFSs have gained attention and some WA, as well as WG, aggregation tools have been developed [51,52]. The weighted averaging operators for TSFSs have been introduced by Ullah et al. [53] and their viability in MADM is demonstrated.

MADM is a rigorous research topic that has been thoroughly studied using WA and WG operators, some distance and similarity measures, and some other techniques as well. Some T-spherical interactive aggregation operators and their applications in MADM are studied [54]. The difficulty of undertaking smart medical device selection using the neutrosophic TOPSIS method [55], as well as the problem of risk management in economics [56], are some examples. To handle strategic planning, a neutrosophic AHP and SWOT method is developed [57] and neutrosophic triangular numbers are utilized in 
MADM problems [58]. The issue of the supplier selection is reduced by using the neutrosophic group ANP-TOPSIS method [59], while the evaluation of e-government websites uses the neutrosophic VIKOR method [60]. Other interesting work on MADM is diverse in its direction [61-73].

Keeping in mind the structure of the TSFS and the importance of describing the membership grades in terms of intervals, we henceforth introduce the concept of the IVTSFS, along with its corresponding WA and WG aggregation operators, such that its viability in MADM is demonstrated. The key goals of this manuscript are as follows:

1. To introduce the idea of the IVTSFS that incorporates the impression of an event with four membership grades in terms of intervals with no limitations.

2. To propose aggregation tools that can be applied to those problems where the IVFS, IVIFS, and IVPFS fail to be applied.

3. To study a MADM problem, utilizing the aggregation operators of IVTSFSs where the selection of the best investment policy is carried out.

4. To prove that the proposed aggregation operators will reduce to match other pre-existing aggregation operators, hence showing their superiority, by using some suitable constraints.

This article is organized as follows: In Section 1, a preamble of pre-existing ideas is studied along with their WA and WG operators. Section 2 will describe some basic concepts and their mutual relations. Section 3 is based on the importance of using interval-valued fuzzy frameworks instead of crisp-valued fuzzy frameworks. Section 4 will be on some basic operations of the sum, product, scalar multiplication, and power operation. The properties of these new operations are also investigated. In Section 5, we will develop the theory of the WA aggregation operators, where we propose the concept of the interval-valued T-spherical fuzzy weighted averaging (IVTSFWA) operator, which is further extended to interval-valued T-spherical fuzzy ordered weighted averaging (IVTSFOWA) and the interval-valued T-spherical fuzzy hybrid averaging (IVTSFHA) operator. The validity of these aggregation operators will be validated using the induction method and their aggregation associated properties are demonstrated. Section 6 will be on the interval-valued T-spherical fuzzy weighted geometric (IVTSFWG), the interval-valued T-spherical fuzzy ordered weighted geometric (IVTSFOWG), and the interval-valued T-spherical fuzzy hybrid geometric (IVTSFHG) operators. Section 7 will be on the construction of a MADM process in the context of IVTSFSs and a MADM problem will be solved using IVTSFWA and IVTSFWG operators. Section 8 will include a comparative study of the proposed operators with the existing ones. The advantages of our proposed work will be discussed in Section 9, followed by the conclusion in Section 10.

\section{Preliminaries}

In this section, the basic definitions of the IVIFS, IVPyFS, IVq-ROPFS, and IVPFS are proposed to analyze their structures. The basic operations of these concepts are also analyzed. Note that $X$ will denote the universe of discourse and $s, i, d$ and $r$ will denote the membership, abstinence, non-membership, and refusal degree of an element, respectively.

Definition 1. [8] An IVq-ROPFS is having the shape $I=\{(s(x), d(x)): x \in X\}$, where $s(x)$ and $d(x)$ are mappings from $X$ to some closed subinterval of $[0,1]$, such that $s(x)=$ $\left[s^{l}(x), s^{u}(x)\right]$ and $d(x)=\left[d^{l}(x), d^{u}(x)\right]$ with a restriction that $0 \leq\left(s^{u}\right)^{q}(x)+\left(d^{u}\right)^{q}(x) \leq$ 1 for some $q \in \mathbb{Z}^{+}$. The term 'hesitancy degree' is denoted and defined by $r(x)=$ $\left(\left[r^{l}(x), r^{u}(x)\right]\right)=\left(\left[\left(1-\left(s^{u}\right)^{q}(x)-\left(d^{u}\right)^{q}\right)^{\frac{1}{q}},\left(\left(1-\left(s^{l}\right)^{q}(x)-\left(d^{l}\right)^{q}\right)^{\frac{1}{q}}\right)\right]\right)$ and the pair $(s(x), d(x))$ $=\left(\left[s^{l}(x), s^{u}(x)\right],\left[d^{l}(x), d^{u}(x)\right]\right)$ is considered as the interval-valued q-rung ortho pair fuzzy number $(I V q-R O P F N)$. 
The following remark will explain the generalization of the IVq-ROPFS over the existing fuzzy structures.

Remark 1. Definition 1 reduces to the definition of:

(1) q-ROPFS if we take $s^{l}(x)=s^{u}(x)$ and $d^{l}(x)=d^{u}(x)$. Yager [6]

(2) IVPyFS if we take $q=2$. Peng and Yang [7]

(3) PyFS if we take $q=2, s^{l}(x)=s^{u}(x)$ and $d^{l}(x)=d^{u}(x)$. Yager [5]

(4) IVIFS if we take $q=1$. Atanassov and Gargov [4]

(5) IFS if we take $q=1, s^{l}(x)=s^{u}(x)$ and $d^{l}(x)=d^{u}(x)$. Atanassov [3]

(6) IVFS if we take $q=1$ and $d^{l}(x)=d^{u}(x)=0$. Gorzalczany [2]

(7) FS if we take $q=1, s^{l}(x)=s^{u}(x)$ Zadeh [1].

Definition 2. [22] A TSFS is having the shape $I=\{(s(x), i(x), d(x)): x \in X\}$, where $s(x), i(x)$, and $d(x)$ are mappings from $X$ to $[0,1]$ with a restriction that $0 \leq s^{q}(x)+i^{q}(x)+d^{q}(x) \leq 1$ for some $q \in \mathbb{Z}^{+}$. The term 'refusal degree' is defined by $r(x)=\left(1-\left(s^{q}(x)+i^{q}(x)+d^{q}(x)\right)\right)^{\frac{1}{q}}$ and the triplet $(s(x), i(x), d(x))$ is considered to be a T-spherical fuzzy number (TSFN).

The generalization of the TSFS is made clear from the following remark:

Remark 2. Definition 2 reduces to the definition of:

(1) $q$-ROPFS if we take $i(x)=0$. Yager [6]

(2) SFS if we take $q=2$. Mahmood et al. [22]

(3) PyFS if we take $q=2$ and $i(x)=0$. Yager [5]

(4) PFS if we take $q=1$. Cuong [17]

(5) IFS if we take $q=1$ and $i(x)=0$. Atanassov [3]

(6) FS if we take $q=1$ and $i(x)=0=d(x)$ Zadeh [1].

The basic operations for two PyFSs were proposed in the following definition:

Definition 3. $[39,40]$ For PyFSs $A, \mathcal{B}$ and for $\lambda>0$, we have:

(1) $A \oplus \mathcal{B}=\left(\sqrt{s_{A}^{2}+s_{\mathcal{B}}^{2}-s_{A}^{2} \cdot s_{\mathcal{B}}^{2}}, d_{A} \cdot d_{\mathcal{B}}\right)$.

(2) $\quad A \otimes \mathcal{B}=\left(s_{A} \cdot s_{\mathcal{B}}, \sqrt{d_{A}^{2}+d_{\mathcal{B}}^{2}-d_{A}^{2} \cdot d_{\mathcal{B}}^{2}}\right)$

(3) $\lambda \cdot A=\left(\sqrt{1-\left(1-s_{A}^{2}\right)^{\lambda}},\left(d_{A}\right)^{\lambda}\right)$.

(4) $A^{\lambda}=\left(\left(s_{A}\right)^{\lambda}, \sqrt{1-\left(1-d_{A}^{2}\right)^{\lambda}}\right)$.

Based on the work discussed in this section, the concept of the IVTSFS is developed and its generalization is proven with the help of some remarks.

\section{The Significance of Interval-Valued Fuzzy Structures}

The idea of representing the membership grade of a FS using an interval was developed by Gorzalczany [2]. Sometimes, the impression of an event cannot be characterized by a crisp number. In such occasions, the concept of the IVFS can be a useful tool. The interval-valued frameworks for the IFS, PyFS, q-ROPFS, and PFS are developed in $[4,7,8,17]$. Now, we discuss the significance of using interval-valued frameworks instead of the ordinary fuzzy framework numerically. 
Consider the following example in which the selection of the best candidate is to be carried out among four candidates $\left(C_{i}, i=1,2,3,4\right)$ based on four attributes $\left(G_{j}, j=1,2,3,4\right)$ under the weight vector $w=(0.22,0.34,0.27,0.17)^{T}$. In this case, the evaluation information of the candidates is in the form of IVIFNs, which are given in Table 1.

Table 1. The decision matrix where $C_{i}$ denote alternatives and $G_{j}$ denote the attributes.

\begin{tabular}{ccccc}
\hline & $G_{1}$ & $G_{2}$ & $G_{3}$ & $G_{4}$ \\
\hline$C_{1}$ & $\left(\begin{array}{l}{[0.2,0.3],} \\
{[0.0,0.5]}\end{array}\right)$ & $\left(\begin{array}{l}{[0.5,0.6],} \\
{[0.1,0.3]}\end{array}\right)$ & $\left(\begin{array}{l}{[0.4,0.5],} \\
{[0.2,0.4]}\end{array}\right)$ & $\left(\begin{array}{l}{[0.7,0.8],} \\
{[0.1,0.2]}\end{array}\right)$ \\
\hline$C_{2}$ & $\left(\begin{array}{l}{[0.6,0.7],} \\
{[0.2,0.3]}\end{array}\right)$ & $\left(\begin{array}{l}{[0.5,0.6],} \\
{[0.0,0.3]}\end{array}\right)$ & $\left(\begin{array}{l}{[0.6,0.7]} \\
{[0.2,0.3]}\end{array}\right)$ & $\left(\begin{array}{l}{[0.6,0.7],} \\
{[0.1,0.2}\end{array}\right)$ \\
\hline \multirow{2}{*}{$C_{3}$} & $\left(\begin{array}{l}{[0.4,0.5],} \\
{[0.3,0.4]}\end{array}\right)$ & $\left(\begin{array}{l}{[0.3,0.6],} \\
{[0.1,0.3]}\end{array}\right)$ & $\left(\begin{array}{l}{[0.5,0.6],} \\
{[0.3,0.4]}\end{array}\right)$ & $\left(\begin{array}{l}{[0.6,0.7],} \\
{[0.1,0.3]}\end{array}\right)$ \\
\hline$C_{4}$ & $\left(\begin{array}{l}{[0.6,0.7],} \\
{[0.2,0.3]}\end{array}\right)$ & $\left(\begin{array}{l}{[0.5,0.7],} \\
{[0.1,0.3]}\end{array}\right)$ & $\left(\begin{array}{l}{[0.7,0.8],} \\
{[0.1,0.2]}\end{array}\right)$ & $\left(\begin{array}{l}{[0.3,0.4],} \\
{[0.1,0.2}\end{array}\right)$ \\
\hline
\end{tabular}

To aggregate the data provided in Table 1, $\mathrm{Xu}$ and Cai [32] developed the following aggregation tool:

$$
\begin{aligned}
& c_{i}=\operatorname{IVIFWA}\left(C_{1}, C_{2}, C_{3} \ldots C_{m}\right) \\
& =\left(\left[1-\prod_{j=1}^{m}\left(1-\left(s_{j}^{l}\right)\right)^{w_{j}}, 1-\prod_{j=1}^{m}\left(1-\left(s_{j}^{u}\right)\right)^{w_{j}}\right],\left[\prod_{j=1}^{m}\left(d_{j}^{l}\right)^{w_{j}}, \prod_{j=1}^{m}\left(d_{j}^{u}\right)^{w_{j}}\right]\right)
\end{aligned}
$$

The aggregated results are:

$$
\begin{aligned}
& c_{1}=\operatorname{IVIFWA}\left(C_{11}, C_{12}, C_{13}, C_{14}\right) \\
& =([0.6235,0.7569],[0.0000,0.3386]) \\
& c_{2}=\operatorname{IVIFWA}\left(C_{21}, C_{22}, C_{23}, C_{24}\right) \\
& =([0.6777,0.8180],[0.0000,0.2800]) \\
& c_{3}=\operatorname{IVIFWA}\left(C_{31}, C_{32}, C_{33}, C_{34}\right) \\
& =([0.5730,0.7745],[0.1713,0.3454]) \\
& c_{4}=\operatorname{IVIFWA}\left(C_{41}, C_{42}, C_{43}, C_{44}\right) \\
& =([0.6493,0.8352],[0.1165,0.2510])
\end{aligned}
$$

The score of IVFN, $I=\left(\left[s^{l}, s^{u}\right],\left[d^{l}, d^{u}\right]\right)$, is defined as [34]:

$$
S C(I)=\frac{1}{2}\left(s^{l}-d^{l}+s^{u}-d^{u}\right)
$$

Using this score's functions, we have:

$$
S C\left(c_{1}\right)=0.5209, S C\left(c_{2}\right)=0.6079, S C\left(c_{3}\right)=0.4154, S C\left(c_{4}\right)=0.5585
$$

Clearly,

$$
S C\left(c_{2}\right)>S C\left(c_{4}\right)>S C\left(c_{1}\right)>S C\left(c_{3}\right)
$$

Hence, $C_{2}$ is the suitable candidate, according to the MADM method based on the IVIFWA operators.

Assigning a crisp value to the membership and non-membership grade of the above problem reduces the information in Table 1 in the context of IFSs. Such information can be aggregated using the 
aggregation operators proposed by $\mathrm{Xu}$ [24]. The decision matrix for the problem discussed above in the context of IFSs is given in Table 2.

Table 2. The decision matrix where $C_{i}$ denote alternatives and $G_{j}$ denote the attributes.

\begin{tabular}{ccccc}
\hline & $G_{1}$ & $G_{2}$ & $G_{3}$ & $G_{4}$ \\
\hline$C_{1}$ & $(0.3,0.5)$ & $(0.6,0.3)$ & $(0.5,0.4)$ & $(0.8,0.2)$ \\
$C_{2}$ & $(0.7,0.3)$ & $(0.6,0.3)$ & $(0.7,0.3)$ & $(0.7,0.2)$ \\
$C_{3}$ & $(0.5,0.4)$ & $(0.6,0.3)$ & $(0.6,0.4)$ & $(0.7,0.3)$ \\
$C_{4}$ & $(0.6,0.2)$ & $(0.5,0.1)$ & $(0.7,0.1)$ & $(0.3,0.1)$ \\
\hline
\end{tabular}

The weighted averaging aggregation operator of the IFSs, proposed by $\mathrm{Xu}$ [24], is given by:

$$
\begin{aligned}
& c_{i}=\operatorname{IFWA}\left(C_{1}, C_{2}, C_{3} \ldots C_{m}\right) \\
& =\left(1-\prod_{j=1}^{m}\left(1-\left(s_{j}^{l}\right)\right)^{w_{j}}, \prod_{j=1}^{m}\left(d_{j}^{l}\right)^{w_{j}}\right) \\
& \quad c_{1}=\operatorname{IFWA}\left(C_{11}, C_{12}, C_{13}, C_{14}\right) \\
& \quad=(0.7569,0.3386) \\
& \quad c_{2}=\operatorname{IFWA}\left(C_{21}, C_{22}, C_{23}, C_{24}\right) \\
& \quad=(0.8180,0.2800) \\
& \quad c_{3}=\operatorname{IFWA}\left(C_{31}, C_{32}, C_{33}, C_{34}\right) \\
& =(0.7745,0.3454) \\
& \quad c_{4}=\operatorname{IFWA}\left(C_{41}, C_{42}, C_{43}, C_{44}\right) \\
& =(0.7489,0.1165)
\end{aligned}
$$

For the ranking of IFN $I$, the score function developed in [24] is given by:

$$
S C(I)=s-d
$$

Using this score function, we have:

$$
S C\left(c_{1}\right)=0.4183, S C\left(c_{2}\right)=0.5380, S C\left(c_{3}\right)=0.4291, S C\left(c_{4}\right)=0.6324
$$

Clearly,

$$
S C\left(c_{4}\right)>S C\left(c_{2}\right)>S C\left(c_{3}\right)>S C\left(c_{1}\right)
$$

Thus, $C_{4}$ is the suitable candidate, according to the MADM method based on the IVIFWA operators.

By analyzing both results, it is observed that the result obtained using the aggregation of the IVIFS is significant compared to the one obtained using the IFS. This shows the importance of using interval-valued fuzzy frameworks over crisp-valued fuzzy frameworks.

The concept of the TSFS developed in [22] is a generalization of the q-ROPFS, SFS, PyFS, PFS, IFS, and FS. Therefore, the interval-valued structure for TSFSs would have a great impact, due to the diverse structure of the TSFS. The concept of the IVTSFS will provide flexibility in assigning the membership grades of the TSFS, in terms of the intervals rather than by a crisp number. Furthermore, as discussed in the first section, there are some situations, such as voting or decision-making, where opinions may involve more than two or three aspects. To deal with such situations, the TSFS has been proven to be useful $[22,23,62]$. Therefore, the idea of TSFSs is further strengthened by the introduction of the IVTSFS. In the next section, the definition of the IVTSFS is proposed and its superiority over the existing fuzzy concepts is shown, with the help of some useful remarks. 


\section{Interval-Valued T-Spherical Fuzzy Set}

This section describes the concept of the IVTSFS and its comparison with pre-existing concepts. We develop the basic operations for IVTSFSs and their properties are investigated. Note that $\operatorname{IVTSFS}(X)$ will denote the set of all of the IVTSFSs.

Definition 4. An IVTSFS has the shape $I=\{(s(x), i(x), d(x)): x \in X\}$, where $s(x), i(x)$, and $d(x)$ are mappings from $X$ to a closed subinterval of $[0,1]$, such that $s(x)=\left[s^{l}(x), s^{u}(x)\right], i(x)=\left[i^{l}(x), i^{u}(x)\right]$, and $d(x)=\left[d^{l}(x), d^{u}(x)\right]$ with a restriction that $0 \leq\left(s^{u}\right)^{q}(x)+\left(i^{u}\right)^{q}(x)+\left(d^{u}\right)^{q}(x) \leq 1$ for some $q \in \mathbb{Z}^{+}$. The term 'refusal degree' is defined by $r(x)=\left(\left[r^{l}(x), r^{u}(x)\right]\right)=$ $\left(\left[\left(1-\left(s^{u}\right)^{q}(x)-\left(i^{u}\right)^{q}(x)-\left(d^{u}\right)^{q}\right)^{\frac{1}{q}},\left(\left(1-\left(s^{l}\right)^{q}(x)-\left(i^{l}\right)^{q}(x)-\left(d^{l}\right)^{q}\right)^{\frac{1}{q}}\right)\right]\right)$ and the pair $(s(x), i(x), d(x))=\left(\left[s^{l}(x), s^{u}(x)\right],\left[i^{l}(x), i^{u}(x)\right],\left[d^{l}(x), d^{u}(x)\right]\right)$ is considered to be the interval-valued T-spherical fuzzy number (IVTSFN).

The existing frameworks will become special cases of the IVTSFS. Consider the following theorem:

Theorem 1. An IVTSFS reduces to:

(1) TSFS: if we consider $s^{l}=s^{u}, i^{l}=i^{u}$ and $d^{l}=d^{u}$ [22].

(2) IVSFS: if we consider $q=2$.

(3) SFS: if we consider $q=2$ and $s^{l}=s^{u}, i^{l}=i^{u}$, and $d^{l}=d^{u}$ [22].

(4) IVPFS: if we consider $q=1$ [17].

(5) PFS: if we consider $q=1$ and $s^{l}=s^{u}, i^{l}=i^{u}$ and $d^{l}=d^{u}$ [17].

(6) IVq-ROPFS: if we consider $i^{l}=i^{u}=0$ [8].

(7) q-ROPFS: if we consider $s^{l}=s^{u}, i^{l}=i^{u}$, and $d^{l}=d^{u}[6]$.

(8) IVPyFS: if we consider $q=2$ and $i^{l}=i^{u}=0$ [7].

(9) PyFS: if we consider $q=2$ and $s^{l}=s^{u}, i^{l}=i^{u}=0$ and $d^{l}=d^{u}[5]$.

(10) IVIFS: if we consider $q=1$ and $i^{l}=i^{u}=0$ [4].

(11) IFS: if we consider $q=1$ and $s^{l}=s^{u}, i^{l}=i^{u}=0$ and $d^{l}=d^{u}$ [3].

(12) IVFS: if we consider $q=1$ and $i^{l}=i^{u}=d^{l}=d^{u}=0$ [2].

(13) FS: if we consider $q=1$ and $s^{l}=s^{u}, i^{l}=i^{u}=0=d^{l}=d^{u}[1]$.

Next, the operations of the sum, product, scalar multiplications, and power operation for IVTSFSs will be introduced. With the help of a remark, it is shown that these operations are generalizations of operations of existing ideas under some restrictions.

Definition 5. For $A, \mathcal{B} \in \operatorname{IVTSFS}(X)$. The operations of the sum, product, scalar multiplications, and power are defined as:

$$
\begin{aligned}
& \text { (1) } \left.\left.\left.\begin{array}{l}
A \oplus \mathcal{B}=\left\{\left(\left[\sqrt[q]{s_{A}^{l} q(x)+s_{\mathcal{B}}^{l} q(x)-s_{A}^{l} q(x) \cdot s_{\mathcal{B}}^{l} q(x)},\right.\right.\right. \\
\sqrt[q]{s_{A}^{u} q(x)+s_{\mathcal{B}}^{u} q(x)-s_{A}^{u} q(x) \cdot s_{\mathcal{B}}^{u} q(x)}
\end{array}\right],\left[i_{A}^{l}(x) \cdot i_{\mathcal{B}}^{l}(x), i_{A}^{u}(x) \cdot i_{\mathcal{B}}^{u}(x)\right],\left[d_{A}^{l}(x) \cdot d_{\mathcal{B}}^{l}(x), d_{A}^{u}(x) \cdot d_{\mathcal{B}}^{u}(x)\right]\right)\right\} \\
& \text { (2) } \quad A \otimes \mathcal{B}=\left\{\left(\left[s_{A}^{l}(x) \cdot s_{\mathcal{B}}^{l}(x), s_{A}^{u}(x) \cdot s_{\mathcal{B}}^{u}(x)\right],\left[i_{A}^{l}(x) \cdot i_{\mathcal{B}}^{l}(x), i_{A}^{u}(x) \cdot i_{\mathcal{B}}^{u}(x)\right],\left[\sqrt[q]{d_{A}^{l} q(x)+d_{\mathcal{B}}^{l} q(x)-d_{A}^{l} q(x) \cdot d_{\mathcal{B}}^{l} q(x)},\right]\right)\right\} \\
& \text { (3) } \left.\quad \lambda A=\left(\left[\sqrt[q]{d_{A}^{u} q(x)+d_{\mathcal{B}}^{u} q(x)-d_{A}^{u} q(x) \cdot d_{\mathcal{B}}^{u} q(x)}\right]\right)\right\} \\
& \text { (4) } \quad A^{\lambda}=\left(\left[\left(s_{A}^{l}\right)^{\lambda},\left(s_{A}^{u}\right)^{\lambda}\right],\left[\left(i_{A}^{l}\right)^{\lambda},\left(i_{A}^{u}\right)^{\lambda}\right],\left[\sqrt[q]{1-\left(1-s_{A}^{u} q\right)^{\lambda}}\right],\left[\left(i_{A}^{l}\right)^{\lambda},\left(i_{A}^{u}\right)^{\lambda}\right],\left[\left(d_{A}^{l}\right)^{\lambda},\left(d_{A}^{u}\right)^{\lambda}\right]\right)
\end{aligned}
$$


Remark 3. The operations in Definition 5 become valid for:

(1) TSFSs: if we consider $s^{l}=s^{u}, i^{l}=i^{u}$, and $d^{l}=d^{u}$.

(2) IVSFSs: if we consider $q=2$.

(3) SFSs: if we consider $q=2$ and $s^{l}=s^{u}, i^{l}=i^{u}$ and $d^{l}=d^{u}$.

(4) IVPFSs: if we consider $q=1$.

(5) PFSs: if we consider $q=1$ and $s^{l}=s^{u}, i^{l}=i^{u}$ and $d^{l}=d^{u}$ [52].

(6) IVq-ROPFSs: if we consider $i^{l}=i^{u}=0$.

(7) q-ROPFSs: if we consider $s^{l}=s^{u}, i^{l}=i^{u}$ and $d^{l}=d^{u}$.

(8) IVPyFSs: if we consider $q=2$ and $i^{l}=i^{u}=0[41,42]$.

(9) PyFSs: if we consider $q=2$ and $s^{l}=s^{u}, i^{l}=i^{u}=0$ and $d^{l}=d^{u}[39,40]$ (Definition 3).

(10) IVIFSs: if we consider $q=1$ and $i^{l}=i^{u}=0[29,30]$.

(11) IFSs: if we consider $q=1$ and $s^{l}=s^{u}, i^{l}=i^{u}=0$ and $d^{l}=d^{u}[24,25]$.

(12) IVFSs: if we consider $q=1$ and $i^{l}=i^{u}=d^{l}=d^{u}=0$.

(13) FSs: if we consider $q=1$ and $s^{l}=s^{u}, i^{l}=i^{u}=0=d^{l}=d^{u}$.

Theorem 2. The following properties hold true for $A, \mathcal{B} \in \operatorname{IVTSFS}(X)$, where $\lambda, \lambda_{1}, \lambda_{3}>0$.

(1) $A \oplus \mathcal{B}=\mathcal{B} \oplus A$

(2) $A \otimes \mathcal{B}=\mathcal{B} \otimes A$

(3) $\lambda(A \oplus \mathcal{B})=\lambda A \oplus \lambda \mathcal{B}$

(4) $\quad(A \otimes \mathcal{B})^{\lambda}=A^{\lambda} \otimes \mathcal{B}^{\lambda}$

(5) $\quad \lambda_{1} A \oplus \lambda_{2} A=\left(\lambda_{1}+\lambda_{2}\right) A$

(6) $A^{\lambda_{1}} \otimes A^{\lambda_{2}}=A^{\lambda_{1}+\lambda_{2}}$

(7) $\left(A^{c}\right)^{\lambda}=(\lambda A)^{c}$

(8) $\lambda\left(A^{c}\right)=\left(A^{\lambda}\right)^{c}$

(9) $\quad A^{c} \oplus \mathcal{B}^{c}=(A \otimes \mathcal{B})^{c}$

(10) $A^{c} \otimes \mathcal{B}^{c}=(A \oplus \mathcal{B})^{c}$

Proof. Trivial.

The ranking of two numbers in fuzzy algebraic structures has a certain importance, especially in MADM problems, medical diagnostics problems, and in pattern recognition. There are several rules defined for the ranking of two numbers of a certain fuzzy algebraic structure, as discussed in Section $1[1,2,38]$. Here, we follow the idea of Joshi and Kumar [38], to define a score function for an IVTSFN.

Definition 6. For an IVTSFN $I=\left(s_{I}(x), i_{I}(x), d_{I}(x)\right)=\left(\left[s^{l}, s^{u}\right],\left[i^{l}, i^{u}\right],\left[d^{l}, d^{u}\right]\right)$, the score function is defined as:

$$
S C(I)=\frac{\left(s^{l}\right)^{q}\left(1-\left(i^{l}\right)^{q}-\left(d^{l}\right)^{q}\right)+\left(s^{u}\right)^{q}\left(\left(1-\left(i^{u}\right)^{n}-\left(d^{u}\right)^{q}\right)\right)}{3} \text { and } \operatorname{SC}(A) \in[0,1]
$$

Remark 4. Using the restrictions stated in Theorem 1, the score value for the TSFS, IVSFS, SFS, IVPFS, and PFS, as well as other fuzzy algebraic structures, can be obtained analogously. 
Example 1. Let $I_{1}=([0.3,0.6],[0.2,0.7],[0.5,0.8])$ and $I_{2}=([0.2,0.4],[0.2,0.3],[0.3,0.8])$ be two IVTSFNs for $q=4$. Then, using the score function defined in Definition 6, we have:

$$
S C\left(I_{1}\right)=0.0227 \text { and } S C\left(I_{2}\right)=0.0066
$$

Clearly, $S C\left(I_{1}\right)>S C\left(I_{2}\right)$. So $I_{1}>I_{2}$.

\section{Averaging Aggregation Operators for Interval-Valued T-Spherical Fuzzy Sets}

In this section, some aggregation operators for IVTSFSs will be proposed. These operators include the IVTSFWA operator, IVTSFOWA operator, and IVTSFHA operator. The validation and basic properties of these aggregation tools will be studied and supported by numerical examples. In our study of aggregation, $w=\left(w_{1}, w_{2}, w_{3}, \ldots w_{m}\right)^{T}$ will denote the weight vector, such that $w_{i} \in[0,1]$ for $i=1,2,3 \ldots m$ and $\sum_{i=1}^{m} w_{i}=1$.

Definition 7. The IVTSFWA operator for IVTSFNs $I_{j}(j=1,2,3 \ldots m)$ is of the form:

$$
\operatorname{IVTSFWA}\left(I_{1}, I_{2}, I_{3} \ldots I_{m}\right)=\sum_{j=1}^{m} w_{j} I_{j}
$$

Theorem 3. The aggregated value of IVTSFNs $I_{j}$, using the IVTSFWA operator, is an IVTSFN which is given as:

$$
\begin{aligned}
& \operatorname{IVTSFWA}\left(I_{1}, I_{2}, I_{3} \ldots I_{m}\right) \\
& =\left(\left[\sqrt[q]{1-\prod_{j=1}^{m}\left(1-\left(s_{j}^{l}\right)^{q}\right)^{w_{j}}}, \sqrt[q]{1-\prod_{j=1}^{m}\left(1-\left(s_{j}^{u}\right)^{q}\right)^{w_{j}}}\right],\left[\prod_{j=1}^{m}\left(i_{j}^{l}\right)^{w_{j}}, \prod_{j=1}^{m}\left(i_{j}^{u}\right)^{w_{j}}\right],\left[\prod_{j=1}^{m}\left(d_{j}^{l}\right)^{w_{j}}, \prod_{j=1}^{m}\left(d_{j}^{u}\right)^{w_{j}}\right]\right)
\end{aligned}
$$

Proof. Following the tradition, this result can be proven using mathematical induction.

For $m=2$.

$$
w_{1} I_{1}=\left(\left[\sqrt[q]{1-\left(1-s_{I_{1}}^{l} q\right)^{w_{1}}}, \sqrt[q]{1-\left(1-s_{I_{1}}^{u q}\right)^{w_{1}}}\right],\left[\left(i_{I_{1}}^{l}\right)^{w_{1}},\left(i_{I_{1}}^{u}\right)^{w_{1}}\right],\left[\left(d_{I_{1}}^{l}\right)^{w_{1}},\left(d_{I_{1}}^{u}\right)^{w_{1}}\right]\right)
$$

and

$$
w_{2} I_{2}=\left(\left[\sqrt[q]{1-\left(1-s_{I_{2}}^{l} q\right)^{w_{2}}}, \sqrt[q]{1-\left(1-s_{I_{2}}^{u q}\right)^{w_{2}}}\right],\left[\left(i_{I_{2}}^{l}\right)^{w_{2}},\left(i_{I_{2}}^{u}\right)^{w_{2}}\right],\left[\left(d_{I_{2}}^{l}\right)^{w_{2}},\left(d_{I_{2}}^{u}\right)^{w_{2}}\right]\right)
$$




$$
\begin{aligned}
& w_{1} I_{1} \oplus w_{2} I_{2}=\left(\begin{array}{c}
\left.\left(\left[\sqrt[q]{1-\left(1-s_{I_{1}}^{l} q\right)^{w_{1}}}, \sqrt[q]{1-\left(1-s_{I_{1}}^{u} q\right)^{w_{1}}}\right],\left[\left(i_{I_{1}}^{l}\right)^{w_{1}},\left(i_{I_{1}}^{u}\right)^{w_{1}}\right],\left[\left(d_{I_{1}}^{l}\right)^{w_{1}},\left(d_{I_{1}}^{u}\right)^{w_{1}}\right]\right) \oplus\right) \\
\left(\left[\sqrt[q]{1-\left(1-s_{I_{2}}^{l} q\right)^{w_{2}}}, \sqrt[q]{1-\left(1-s_{I_{2}}^{u} q\right)^{w_{2}}}\right],\left[\left(i_{I_{2}}^{l}\right)^{w_{2}},\left(i_{I_{2}}^{u}\right)^{w_{2}}\right],\left[\left(d_{I_{2}}^{l}\right)^{w_{2}},\left(d_{I_{2}}^{u}\right)^{w_{2}}\right]\right)
\end{array}\right)
\end{aligned}
$$

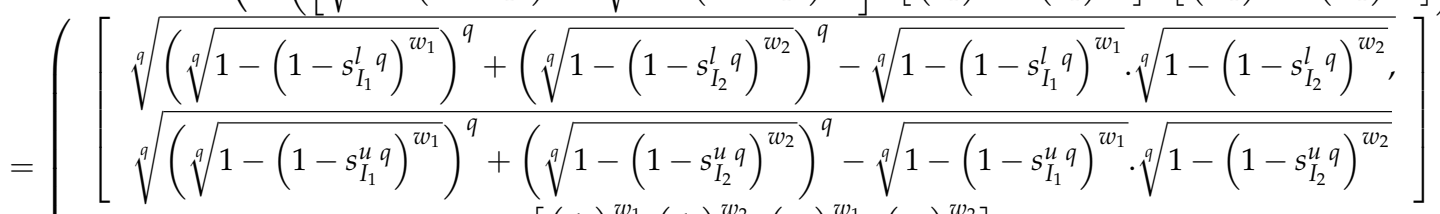

$$
\begin{aligned}
& {\left[\left(i_{I_{1}}^{l}\right)^{w_{1}} \cdot\left(i_{I_{2}}^{l}\right)^{w_{2}},\left(i_{I_{1}}^{u}\right)^{w_{1}} \cdot\left(i_{I_{2}}^{u}\right)^{w_{2}}\right]} \\
& {\left[\left(d_{I_{1}}^{l}\right)^{w_{1}} \cdot\left(d_{I_{2}}^{l}\right)^{w_{2}},\left(d_{I_{1}}^{u}\right)^{w_{1}} \cdot\left(d_{I_{2}}^{u}\right)^{w_{2}}\right.} \\
& =\left(\begin{array}{c}
{\left[\sqrt[q]{1-\left(1-s_{I_{1}}^{l} q\right)^{w_{1}} \cdot\left(1-s_{I_{2}}^{l} q\right)^{w_{2}}}, \sqrt[q]{1-\left(1-s_{I_{1}}^{u} q\right)^{w_{1}} \cdot\left(1-s_{I_{2}}^{u} q\right)^{w_{2}}}\right]} \\
{\left[\left(i_{I_{1}}^{l}\right)^{w_{1}} \cdot\left(i_{I_{2}}^{l}\right)^{w_{2}},\left(i_{I_{1}}^{u}\right)^{w_{1}} \cdot\left(i_{I_{2}}^{u}\right)^{w_{2}}\right]} \\
{\left[\left(d_{I_{1}}^{l}\right)^{w_{1}} \cdot\left(d_{I_{2}}^{l}\right)^{w_{W_{2}}},\left(d_{I_{1}}^{u}\right)^{w_{1}} \cdot\left(d_{I_{2}}^{u}\right)^{w_{2}}\right]}
\end{array}\right) \\
& =\left(\left[\sqrt[q]{\left.1-\prod_{j=1}^{2}\left(1-s_{I_{j}}^{l}\right)^{q}\right)^{w_{j}}}, \sqrt[q]{1-\prod_{j=1}^{2}\left(1-s_{I_{j}}^{u q}\right)^{w_{j}}}\right],\left[\prod_{j=1}^{2}\left(i_{I_{j}}^{l} q\right)^{w_{j}}, \prod_{j=1}^{2}\left(i_{I_{j}}^{u q}\right)^{w_{j}}\right],\left[\prod_{j=1}^{2}\left(d_{I_{j}}^{l} q\right)^{w_{j}}, \prod_{j=1}^{2}\left(d_{I_{j}}^{u q}\right)^{w_{j}}\right]\right)
\end{aligned}
$$

Hence, for $m=2$, the result is true.

Supposedly, we claim that the result is valid for $m=k$, i.e.,

$$
\begin{aligned}
& \operatorname{IVTSFWA~}\left(I_{1}, I_{2}, I_{3} \ldots I_{k}\right) \\
& =\left(\left[\sqrt[q]{1-\prod_{j=1}^{k}\left(1-s_{j}^{l q}\right)^{w_{j}}}, \sqrt[q]{1-\prod_{j=1}^{k}\left(1-s_{j}^{u q}\right)^{w_{j}}}\right],\left[\prod_{j=1}^{k}\left(i_{I_{j}}^{l_{j} q}\right)^{w_{j}}, \prod_{j=1}^{k}\left(i_{I_{j}}^{u q}\right)^{w_{j}}\right],\left[\prod_{j=1}^{k}\left(d_{I_{j}}^{l} q\right)^{w_{j}}, \prod_{j=1}^{k}\left(d_{I_{j}}^{u q}\right)^{w_{j}}\right]\right)
\end{aligned}
$$

To prove the result for $m=k+1$, consider:

$$
\begin{aligned}
& \operatorname{IVTSFWA}\left(I_{1}, I_{2}, I_{3} \ldots I_{k}, I_{k+1}\right)=\sum_{j=1}^{k+1} w_{j} I_{j}=\sum_{j=1}^{k} w_{j} I_{j} \oplus w_{k+1} I_{k+1} \\
& =\left(\begin{array}{c}
\left(\left[\sqrt[q]{1-\prod_{j=1}^{k}\left(1-s_{j}^{l q}\right)^{w_{j}}}, \sqrt[q]{\left.1-\prod_{j=1}^{k}\left(1-s_{j}^{u q}\right)^{w_{j}}\right]},\left[\prod_{j=1}^{k}\left(i_{I_{j}}^{l} q\right)^{w_{j}}, \prod_{j=1}^{k}\left(i_{I_{j}}^{u q}\right)^{w_{j}}\right],\left[\prod_{j=1}^{k}\left(d_{I_{j}}^{l} q\right)^{w_{j}}, \prod_{j=1}^{k}\left(d_{I_{j}}^{u q}\right)^{w_{j}}\right]\right) \oplus\right. \\
\left(\left[\sqrt[q]{1-\left(1-s_{k+1}^{l} q\right)^{w_{k+1}}}, \sqrt[q]{1-\left(1-s_{k+1}^{u}\right)^{w_{k+1}}}\right],\left[\left(i_{I_{k+1}}^{l} q\right)^{w_{k+1}},\left(i_{I_{k+1}^{u}}^{u} q\right)^{w_{k+1}}\right],\left[\left(d_{I_{k+1}}^{l} q\right)^{w_{k+1}},\left(d_{I_{k+1}}^{u}\right)^{w_{k+1}}\right]\right)
\end{array}\right)
\end{aligned}
$$

Finally, we get:

$$
\begin{aligned}
& \operatorname{IVTSFWA}\left(I_{1}, I_{2}, I_{3} \ldots I_{k}, I_{k+1}\right) \\
& =\left(\left[\sqrt[q]{1-\prod_{j=1}^{k+1}\left(1-s_{j}^{l q}\right)^{w_{j}}}, \sqrt[q]{1-\prod_{j=1}^{k+1}\left(1-s_{j}^{u q}\right)^{w_{j}}}\right],\left[\prod_{j=1}^{k+1}\left(i_{I_{j}}^{l^{q} q}\right)^{w_{j}}, \prod_{j=1}^{k+1}\left(i_{I_{j}}^{u q}\right)^{w_{j}}\right],\left[\prod_{j=1}^{k+1}\left(d_{I_{j}}^{l} q\right)^{w_{j}}, \prod_{j=1}^{k+1}\left(d_{I_{j}}^{u q}\right)^{w_{j}}\right]\right)
\end{aligned}
$$

Hence, the result is valid for $m=k+1$. So, using the induction method, it is proven that the result is valid for all $m \in \mathbb{Z}^{+}$. In the following theorem, we state that the IVTSFWA operator satisfies the basic characteristics of an aggregation operator.

Theorem 4. For the IVTSFWA operator, the following properties hold true:

\section{(1) Idempotency:}

If $I_{j}=I$ for all $j=1,2,3, \ldots m$, then:

$$
\operatorname{IVTSFWA}\left(I_{1}, I_{2}, I_{3} \ldots I_{m}\right)=I
$$


Proof. Let $I_{j}=I=\left(\left[s^{l}, s^{u}\right],\left[i^{l}, i^{u}\right],\left[d^{l}, d^{u}\right]\right)$ for all $j$, then:

$$
\begin{aligned}
& \operatorname{IVTSFWA}(I, I, I \ldots I) \\
& =\left(\left[\sqrt[q]{1-\prod_{j=1}^{m}\left(1-\left(s^{l}\right)^{q}\right)^{w_{j}}}, \sqrt[q]{1-\prod_{j=1}^{m}\left(1-\left(s^{u}\right)^{q}\right)^{w_{j}}}\right],\left[\prod_{j=1}^{m}\left(i^{l}\right)^{w_{j}}, \prod_{j=1}^{m}\left(i^{u}\right)^{w_{j}}\right],\left[\prod_{j=1}^{m}\left(d^{l}\right)^{w_{j}}, \prod_{j=1}^{m}\left(d^{u}\right)^{w_{j}}\right]\right)
\end{aligned}
$$

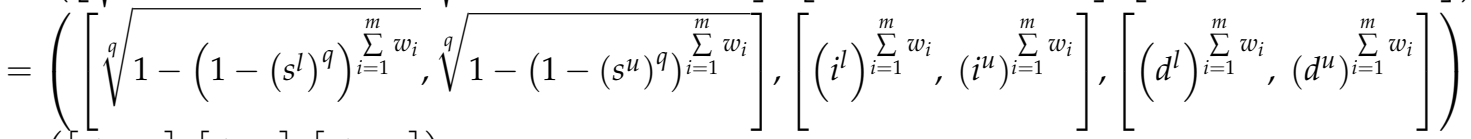

$$
\begin{aligned}
& =\left(\left[s^{l}, s^{u}\right],\left[i^{l}, i^{u}\right],\left[d^{l}, d^{u}\right]\right)=I
\end{aligned}
$$

(2) Boundedness:

If $I^{-}=\min _{j}\left(s_{j}, i_{j} d_{j}\right)$ and $I^{+}=\max _{j}\left(s_{j}, i_{j} d_{j}\right)$ are the least and greatest IVTSFNs, then:

$$
I^{-} \leq \operatorname{IVTSFWA}\left(I_{1}, I_{2}, I_{3} \ldots I_{m}\right) \leq I^{+}
$$

Proof. Straightforward.

(3) Monotonicity:

For the two collections of IVTSFNs, $I_{j}$ and $P_{j}$. If $I_{j} \leq P_{j} \forall j$, then:

$$
\operatorname{IVTSFWA}\left(I_{1}, I_{2}, I_{3} \ldots I_{m}\right) \leq \operatorname{IVTSFWA}\left(P_{1}, P_{2}, P_{3} \ldots P_{m}\right)
$$

Proof. As

$$
\operatorname{IVTSFWA}\left(I_{1}, I_{2}, I_{3} \ldots I_{m}\right)=w_{1} I_{1}+w_{2} I_{2}+w_{3} I_{3}+\ldots w_{m} I_{m}
$$

and

$$
\operatorname{IVTSFWA}\left(P_{1}, P_{2}, P_{3} \ldots P_{m}\right)=w_{1} P_{1}+w_{2} P_{2}+w_{3} P_{3}+\ldots w_{m} P_{m}
$$

As $I_{j} \leq P_{j} \forall j$. So,

$$
\operatorname{IVTSFWA}\left(I_{1}, I_{2}, I_{3} \ldots I_{m}\right) \leq \operatorname{IVTSFWA}\left(P_{1}, P_{2}, P_{3} \ldots P_{m}\right)
$$

In MADM problems, sometimes the ordered position of the information has importance and needs to be weighted. For this reason, the concept of the IVTSFOWA operator is proposed.

Definition 8. The IVTSFOWA operator for IVTSFNs $I_{j}(j=1,2,3 \ldots m)$ is of the form:

$$
\operatorname{IVTSFOWA}\left(I_{1}, I_{2}, I_{3} \ldots I_{m}\right)=\sum_{j=1}^{m} w_{j} I_{\alpha(j)}
$$

where $I_{\alpha(j)}$ denotes the $j^{\text {th }}$ largest value of $I_{j}$.

Theorem 5. The aggregated value of the IVTSFNs, $I_{j}$, using an IVTSFOWA operator, is an IVTSFN and is given as:

$$
\begin{aligned}
& \operatorname{IVTSFOWA}\left(I_{1}, I_{2}, I_{3} \ldots I_{m}\right) \\
& \left.=\left(\left[\sqrt[q]{1-\prod_{j=1}^{m}\left(1-\left(s_{\alpha(j)}^{l}\right)^{q}\right)^{w_{j}}}, \sqrt[q]{1-\prod_{j=1}^{m}\left(1-\left(s_{\alpha(j)}^{u}\right)^{q}\right)^{w_{j}}}\right],\left[\prod_{j=1}^{m}\left(i_{\alpha(j)}^{l}\right)^{w_{j}}, \prod_{j=1}^{m}\left(i_{\alpha(j)}^{l}\right)\right)^{w_{j}}\right],\left[\prod_{j=1}^{m}\left(d_{\alpha(j)}^{l}\right)^{w_{j}}, \prod_{j=1}^{m}\left(d_{\alpha(j)}^{l}\right)^{w_{j}}\right]\right)
\end{aligned}
$$


Proof. This result can analogously be proven, like Theorem 3.

Example 2. Let $I_{1}=([0.3,0.6],[0.2,0.7],[0.5,0.8]), I_{2}=([0.2,0.4],[0.2,0.6],[0.3,0.8]), I_{3}=$ $([0.3,0.3],[0.4,0.5],[0.7,0.8])$, and $I_{4}=([0.1,0.5],[0.2,0.2],[0.3,0.5])$ be the four IVTSFNs for $q=4$ and let $w=(0.22,0.34,0.27,0.17)^{T}$ be the weight vector. Then, using the score function defined in Definition 6 , we have $S C\left(I_{1}\right)=0.0227, S C\left(I_{2}\right)=0.0055, S C\left(I_{3}\right)=0.0089$, and $S C\left(I_{4}\right)=0.0066$. So, the IVTSFNs are ranked as: $I_{1}>I_{3}>I_{4}>I_{2}$ and we get:

$$
\begin{aligned}
I_{\alpha(1)} & =([0.3,0.6],[0.2,0.7],[0.5,0.8]) \\
I_{\alpha(2)} & =([0.3,0.3],[0.4,0.5],[0.7,0.8]) \\
I_{\alpha(3)} & =([0.1,0.5],[0.2,0.2],[0.3,0.5]) \\
I_{\alpha(4)} & =([0.2,0.4],[0.2,0.6],[0.3,0.8])
\end{aligned}
$$

Thus, the aggregated value of $I_{1}, I_{2}, I_{3}$, and $I_{4}$, using the IVTSFOWA operator, is given by:

$$
\begin{gathered}
\operatorname{IVTSFOWA}\left(I_{1}, I_{3}, I_{4}, I_{2}\right) \\
=([0.2691,0.4573],[0.2532,0.4485],[0.3994,0.8000]) .
\end{gathered}
$$

As discussed, whenever the ordered position of IVTSFNs is necessary, we use the IVTSFOWA operator. If the importance of their argument also needs to be weighted, then we develop the IVTSFHA operator.

Definition 9. The IVTSFHA operator for the IVTSFN $I_{j}(j=1,2,3 \ldots m)$ is of the form:

$$
\operatorname{IVTSFHA}\left(I_{1}, I_{2}, I_{3} \ldots I_{m}\right)=\sum_{j=1}^{m} w_{j} \dot{I}_{\alpha(j)}
$$

where $\dot{I}_{j}$ can be computed as $\dot{I}_{j}=m \mathfrak{w} I_{j}, \dot{I}_{\alpha(j)}$ denotes the $j^{\text {th }}$ largest value of $\dot{I}_{j}$, and further $\mathfrak{w}=$ $\left(\mathfrak{w}_{1}, \mathfrak{w}_{2}, \mathfrak{w}_{3} \ldots \mathfrak{w}_{n}\right)^{T}$ is the weight vector of $I_{j}$.

Theorem 6. The aggregated value of the IVTSFNs $I_{j}$, using the IVTSFHA operator, is an IVTSFN and is given as:

$$
\begin{aligned}
& \operatorname{IVTSFHA}\left(I_{1}, I_{2}, I_{3} \ldots I_{m}\right) \\
& =\left(\left[\sqrt[q]{1-\prod_{j=1}^{m}\left(1-\left(\dot{s}_{\alpha(j)}^{l}\right)^{q}\right)^{w_{j}}}, \sqrt[q]{1-\prod_{j=1}^{m}\left(1-\left(\dot{s}_{\alpha(j)}^{u}\right)^{q}\right)^{w_{j}}}\right],\left[\prod_{j=1}^{m}\left(i_{\alpha(j)}^{l}\right)^{w_{j}}, \prod_{j=1}^{m}\left(i_{\alpha(j)}^{l}\right)^{w_{j}}\right],\left[\prod_{j=1}^{m}\left(\dot{d}_{\alpha(j)}^{l}\right)^{w_{j}}, \prod_{j=1}^{m}\left(\dot{d}_{\alpha(j)}^{l}\right)^{w_{j}}\right]\right)
\end{aligned}
$$

Proof. This result can analogously be proven, similarly to the proof of Theorem 3 .

Example 3. Let $I_{1}=([0.3,0.6],[0.2,0.7],[0.5,0.8]), I_{2}=([0.2,0.4],[0.2,0.6],[0.3,0.8]), I_{3}=$ $([0.3,0.3],[0.4,0.5],[0.7,0.8])$, and $I_{4}=([0.1,0.5],[0.2,0.2],[0.3,0.5])$ be the four IVTSFNs for $q=4$ and let $\omega=(0.22,0.34,0.27,0.17)^{T}$ be the weight vector of the IVTSFNs. Furthermore, let $w=(0.2,0.1,0.3,0.4)^{T}$ be the aggregated associated weight vector. Then, using $\dot{I}_{j}=m \mathfrak{w} I_{j}$, we have:

$$
\begin{aligned}
& \dot{I}_{1}=m \mathfrak{w} I_{1} \\
& =\left(\left[\sqrt[4]{1-\left(1-(0.3)^{4}\right)^{4 \times 0.22}}, \sqrt[4]{1-\left(1-(0.6)^{4}\right)^{4 \times 0.22}}\right],\left[(0.2)^{4 \times 0.22},(0.7)^{4 \times 0.22}\right],\left[(0.5)^{4 \times 0.22},(0.8)^{4 \times 0.22}\right]\right) \\
& =([0.3466,0.6379],[0.0004,0.0528],[0.0138,0.0901])
\end{aligned}
$$


Similarly,

$$
\begin{aligned}
& \dot{I}_{2}=m \mathfrak{w} I_{2}=([0.1120,0.2876],[0.0005,0.0441],[0.0028,0.1393]) \\
& \dot{I}_{3}=m \mathfrak{w} I_{3}=([0.2725,0.2725],[0.0069,0.0069],[0.0169,0.1106]) \\
& \dot{I}_{4}=m \mathfrak{w} I_{4}=([0.3347,0.5363],[0.0003,0.0014],[0.0014,0.0696])
\end{aligned}
$$

Now, using the score function defined in Definition 6, we have $S C\left(\dot{I}_{1}\right)=0.5240, \operatorname{SC}\left(\dot{I}_{2}\right)=0.1900$, $S C\left(\dot{I}_{3}\right)=0.3461$, and $S C\left(\dot{I}_{4}\right)=0.5003$. So, the IVTSFNs $\dot{I}_{j}$ are ranked as:

$$
I_{1}>I_{4}>I_{3}>I_{2}
$$

And we get:

$$
\begin{aligned}
& \dot{I}_{\alpha(1)}=([0.3466,0.6379],[0.0004,0.0528],[0.0138,0.0901]) \\
& \dot{I}_{\alpha(2)}=([0.3347,0.5363],[0.0003,0.0014],[0.0014,0.0696]) \\
& \dot{I}_{\alpha(3)}=([0.2725,0.2725],[0.0069,0.0069],[0.0169,0.1106]) \\
& \dot{I}_{\alpha(4)}=([0.1120,0.2876],[0.0005,0.0441],[0.0028,0.1393])
\end{aligned}
$$

Now, the aggregated value of $\dot{I}_{1}, \dot{I}_{2}, \dot{I}_{3}$, and $\dot{I}_{4}$, using the IVTSFHA operator, is given by:

$$
\operatorname{IVTSFHA}\left(\dot{I}_{1}, \dot{I}_{4}, \dot{I}_{3}, \dot{I}_{1}\right)=([0.3129,0.5153],[0.0008,0.0066],[0.0050,0.0903])
$$

Theorem 7. If $w=\left(\frac{1}{m}, \frac{1}{m}, \frac{1}{m}, \ldots \frac{1}{m}\right)^{T}$, then the IVTSFHA operator becomes the IVTSFWA operator.

Proof. As $\dot{I}_{j}=m \mathfrak{w}_{j} I_{j}$ and $w=\left(\frac{1}{m}, \frac{1}{m}, \frac{1}{m}, \ldots \frac{1}{m}\right)^{T}$. So $w_{j} \dot{I}_{j}=\mathfrak{w}_{j} I_{j}$ and

$$
\operatorname{IVTSFHA}\left(I_{1}, I_{2}, I_{3} \ldots I_{m}\right)=\sum_{j=1}^{m} w_{j} \dot{I}_{\sigma(j)}=\sum_{j=1}^{m} \mathfrak{w}_{j} I_{j}=\operatorname{IVTSFWA}\left(I_{1}, I_{2}, I_{3} \ldots I_{m}\right)
$$

\section{Geometric Aggregation Operators for Interval-Valued T-Spherical Fuzzy Sets:}

In this section, some geometric aggregation operators for IVTSFSs are proposed. These operators include the IVTSFWG operator, IVTSFOWG operator, and IVTSFHG operator. The basic properties of these aggregation tools will be studied and supported by numerical examples.

Definition 10. The IVTSFWG operator for the IVTSFNs $I_{j}(j=1,2,3 \ldots m)$ is of the form:

$$
\operatorname{IVTSFWG}\left(I_{1}, I_{2}, I_{3} \ldots I_{m}\right)=\prod_{j=1}^{m} I_{j}^{w_{j}}
$$


Theorem 8. The aggregated value of the IVTSFNs $I_{j}$, using the IVTSFWG operator, is an IVTSFN and is given as:

$$
\begin{aligned}
& \operatorname{IVTSFWG}\left(I_{1}, I_{2}, I_{3} \ldots I_{m}\right) \\
& =\left(\left[\prod_{j=1}^{m}\left(s_{j}^{l}\right)^{w_{j}}, \prod_{j=1}^{m}\left(s_{j}^{u}\right)^{w_{j}}\right],\left[\prod_{j=1}^{m}\left(i_{j}^{l}\right)^{w_{j}}, \prod_{j=1}^{m}\left(i_{j}^{u}\right)^{w_{j}}\right],\left[\sqrt[q]{1-\prod_{j=1}^{m}\left(1-\left(d_{j}^{l}\right)^{q}\right)^{w_{j}}}, \sqrt[q]{1-\prod_{j=1}^{m}\left(1-\left(d_{j}^{u}\right)^{q}\right)^{w_{j}}}\right]\right)
\end{aligned}
$$

Proof. Following the tradition, this result can be proven using mathematical induction.

For $m=2$.

$$
I_{1}^{w_{1}}=\left(\left[\left(s_{I_{1}}^{l}\right)^{w_{1}},\left(s_{I_{1}}^{u}\right)^{w_{1}}\right],\left[\left(i_{I_{1}}^{l}\right)^{w_{1}},\left(i_{I_{1}}^{u}\right)^{w_{1}}\right],\left[\sqrt[q]{1-\left(1-d_{I_{1}}^{l} q\right)^{w_{1}}}, \sqrt[q]{1-\left(1-d_{I_{1}}^{u} q\right)^{w_{1}}}\right]\right)
$$

and

$$
\begin{aligned}
& I_{2}^{w_{2}}=\left(\left[\left(s_{I_{2}}^{l}\right)^{w_{2}},\left(s_{I_{2}}^{u}\right)^{w_{2}}\right],\left[\left(i_{I_{2}}^{l}\right)^{w_{2}},\left(i_{I_{2}}^{u}\right)^{w_{2}}\right],\left[\sqrt[q]{1-\left(1-d_{I_{2}}^{l} q\right)^{w_{2}}}, \sqrt[q]{1-\left(1-d_{I_{2}}^{u q}\right)^{w_{2}}}\right]\right) \\
& I_{1}^{w_{1}} \otimes I_{2}^{w_{2}}=\left(\begin{array}{c}
\left(\left[\left(s_{I_{1}}^{l}\right)^{w_{1}},\left(s_{I_{1}}^{u}\right)^{w_{1}}\right],\left[\left(i_{I_{1}}^{l}\right)^{w_{1}},\left(i_{I_{1}}^{u}\right)^{w_{1}}\right],\left[\sqrt[q]{1-\left(1-d_{I_{1}}^{l} q\right)^{w_{1}}}, \sqrt[q]{1-\left(1-d_{I_{1}}^{u} q\right)^{w_{1}}}\right]\right) \otimes \\
\left(\left[\left(s_{I_{2}}^{l}\right)^{w_{2}},\left(s_{I_{2}}^{u}\right)^{w_{2}}\right],\left[\left(i_{I_{2}}^{l}\right)^{w_{2}},\left(i_{I_{2}}^{u}\right)^{w_{2}}\right],\left[\sqrt[q]{1-\left(1-d_{I_{2}}^{l} q\right)^{w_{2}}}, \sqrt[q]{1-\left(1-d_{I_{2}}^{u} q\right)^{w_{2}}}\right]\right)
\end{array}\right)
\end{aligned}
$$

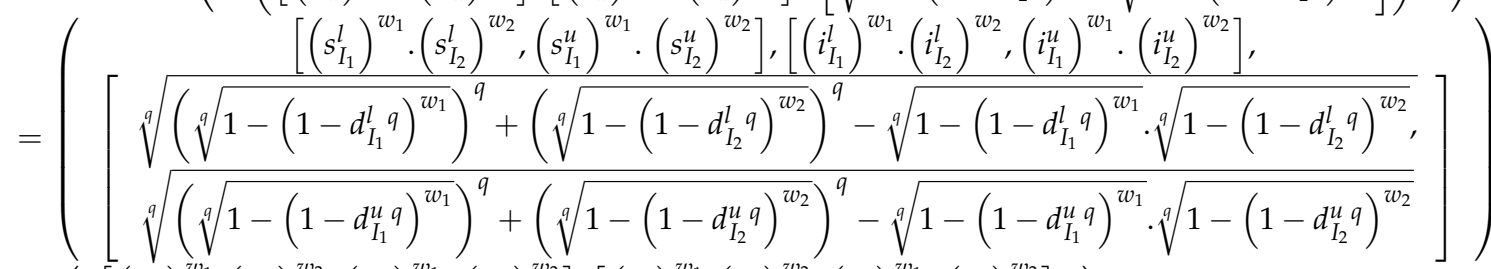

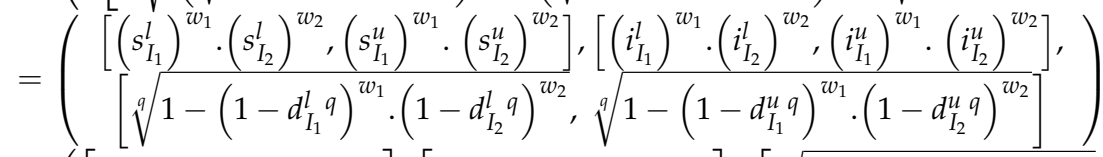

$$
\begin{aligned}
& =\left(\left[\prod_{j=1}^{2}\left(s_{j}^{l}\right)^{w_{j}}, \prod_{j=1}^{2}\left(s_{j}^{u}\right)^{w_{j}}\right],\left[\prod_{j=1}^{2}\left(i_{j}^{l}\right)^{w_{j}}, \prod_{j=1}^{2}\left(i_{j}^{u}\right)^{w_{j}}\right],\left[\sqrt[q]{1-\prod_{j=1}^{2}\left(1-\left(d_{j}^{l}\right)^{q}\right)^{w_{j}}}, \sqrt[q]{1-\prod_{j=1}^{2}\left(1-\left(d_{j}^{u}\right)^{q}\right)^{w_{j}}}\right]\right)
\end{aligned}
$$

Hence, for $m=2$, the result is true.

Based on this assumption, we claim that the result is valid for $m=k$ i.e.,

$$
\begin{aligned}
& \operatorname{IVTSFWG}\left(I_{1}, I_{2}, I_{3} \ldots I_{k}\right) \\
& =\left(\left[\prod_{j=1}^{k}\left(s_{j}^{l}\right)^{w_{j}}, \prod_{j=1}^{k}\left(s_{j}^{u}\right)^{w_{j}}\right],\left[\prod_{j=1}^{k}\left(i_{j}^{l}\right)^{w_{j}}, \prod_{j=1}^{k}\left(i_{j}^{u}\right)^{w_{j}}\right],\left[\sqrt[q]{1-\prod_{j=1}^{k}\left(1-\left(d_{j}^{l}\right)^{q}\right)^{w_{j}}}, \sqrt[q]{1-\prod_{j=1}^{k}\left(1-\left(d_{j}^{u}\right)^{q}\right)^{w_{j}}}\right]\right)
\end{aligned}
$$

To prove the result for $m=k+1$, consider:

$$
\begin{aligned}
& \operatorname{IVTSFWG}\left(I_{1}, I_{2}, I_{3} \ldots I_{k}, I_{k+1}\right)=\prod_{j=1}^{k+1} I_{j}^{w_{j}}=\prod_{j=1}^{k} I_{j}^{w_{j}} \otimes I_{k+1}^{w_{k+1}} \\
& =\left(\begin{array}{c}
\left(\left[\prod_{j=1}^{k}\left(s_{j}^{l}\right)^{w_{j}}, \prod_{j=1}^{k}\left(s_{j}^{u}\right)^{w_{j}}\right],\left[\prod_{j=1}^{k}\left(i_{j}^{l}\right)^{w_{j}}, \prod_{j=1}^{k}\left(i_{j}^{u}\right)^{w_{j}}\right],\left[\sqrt[q]{1-\prod_{j=1}^{k}\left(1-\left(d_{j}^{l}\right)^{q}\right)^{w_{j}}}, \sqrt[q]{1-\prod_{j=1}^{k}\left(1-\left(d_{j}^{u}\right)^{q}\right)^{w_{j}}}\right]\right) \otimes \\
\left(\left[\left(s_{I_{k+1}}^{l}\right)^{w_{k+1}},\left(s_{I_{k+1}}^{u}\right)^{w_{k+1}}\right],\left[\left(i_{I_{k+1}}^{l}\right)^{w_{k+1}},\left(i_{I_{k+1}}^{u}\right)^{w_{k+1}}\right],\left[\sqrt[q]{1-\left(1-d_{I_{k+1}}^{l}\right)^{q} w_{k+1}}, \sqrt[q]{1-\left(1-d_{I_{k+1}}^{u}\right)^{w_{k+1}}}\right]\right.
\end{array}\right)
\end{aligned}
$$


Finally, we get:

$$
\begin{aligned}
& \operatorname{IVTSFWG}\left(I_{1}, I_{2}, I_{3} \ldots I_{k}, I_{k+1}\right) \\
& =\left(\left[\prod_{j=1}^{k+1}\left(s_{j}^{l}\right)^{w_{j}}, \prod_{j=1}^{k+1}\left(s_{j}^{u}\right)^{w_{j}}\right],\left[\prod_{j=1}^{k+1}\left(i_{j}^{l}\right)^{w_{j}}, \prod_{j=1}^{k+1}\left(i_{j}^{u}\right)^{w_{j}}\right],\left[\sqrt[q]{1-\prod_{j=1}^{k+1}\left(1-\left(d_{j}^{l}\right)^{q}\right)^{w_{j}}}, \sqrt[q]{1-\prod_{j=1}^{k+1}\left(1-\left(d_{j}^{u}\right)^{q}\right)^{w_{j}}}\right]\right)
\end{aligned}
$$

Hence, the result is valid for $m=k+1$. Consequently, using the induction method, it is proven that the result is valid for all $m \in \mathbb{Z}^{+}$.

In the following theorem, we state that the IVTSFWG operator satisfies the basic characteristics of aggregation operators.

Theorem 9. For an IVTSFWG operator, the following properties hold true.

\section{(1) Idempotency:}

If $I_{j}=I$ for all $j=1,2,3, \ldots m$, then:

$$
\operatorname{IVTSFWG}\left(I_{1}, I_{2}, I_{3} \ldots I_{m}\right)=I
$$

\section{(2) Boundedness:}

If $I^{-}=\min _{j}\left(s_{j}, i_{j} d_{j}\right)$ and $I^{+}=\max _{j}\left(s_{j}, i_{j} d_{j}\right)$ are the least and greatest IVTSFNs, then:

$$
I^{-} \leq \operatorname{IVTSFWG}\left(I_{1}, I_{2}, I_{3} \ldots I_{m}\right) \leq I^{+}
$$

\section{(3) Monotonicity:}

For the two collections of IVTSFNs, $I_{j}$ and $P_{j}$, if $I_{j} \leq P_{j} \forall j$ then:

$$
\operatorname{IVTSFWA}\left(I_{1}, I_{2}, I_{3} \ldots I_{m}\right) \leq \operatorname{IVTSFWA}\left(P_{1}, P_{2}, P_{3} \ldots P_{m}\right)
$$

These results can be proven analogously.

We have developed the IVTSFOWA operator to handle situations where the ordered position of the information is important. Now, the concept of the IVTSFOWG operator is proposed.

Definition 11. The IVTSFOWG operator for IVTSFNs $I_{j}(j=1,2,3 \ldots m)$ is of the form:

$$
\operatorname{IVTSFOWG}\left(I_{1}, I_{2}, I_{3} \ldots I_{m}\right)=\prod_{j=1}^{m} I_{\alpha(j)}^{w_{j}}
$$

where $I_{\alpha(j)}$ denotes the $j^{\text {th }}$ largest value of $I_{j}$.

Theorem 10. The aggregated value of the IVTSFNs $I_{j}$, using the IVTSFOWG operator, is an IVTSFN and is given as:

$$
\begin{aligned}
& \operatorname{IVTSFOWG}\left(I_{1}, I_{2}, I_{3} \ldots I_{m}\right) \\
& =\left(\left[\prod_{j=1}^{m}\left(s_{\alpha(j)}^{l}\right)^{w_{j}}, \prod_{j=1}^{m}\left(s_{\alpha(j)}^{u}\right)^{w_{j}}\right],\left[\prod_{j=1}^{m}\left(i_{\alpha(j)}^{l}\right)^{w_{j}}, \prod_{j=1}^{m}\left(i_{\alpha(j)}^{u}\right)^{w_{j}}\right],\left[\sqrt[q]{1-\prod_{j=1}^{m}\left(1-\left(d_{\alpha(j)}^{l}\right)^{q}\right)^{w_{j}}}, \sqrt[q]{1-\prod_{j=1}^{m}\left(1-\left(d_{\alpha(j)}^{u}\right)^{q}\right)^{w_{j}}}\right]\right)
\end{aligned}
$$

Example 4. Let $I_{1}=([0.3,0.6],[0.2,0.7],[0.5,0.8]), I_{2}=([0.2,0.4],[0.2,0.6],[0.3,0.8]), I_{3}=$ $([0.3,0.3],[0.4,0.5],[0.7,0.8])$, and $I_{4}=([0.1,0.5],[0.2,0.2],[0.3,0.5])$ be the four IVTSFNs for $q=4$ and let $w=(0.22,0.34,0.27,0.17)^{T}$ be the weight vector. Then, using the score function defined in Definition 
6, we have $S C\left(I_{1}\right)=0.0227, S C\left(I_{2}\right)=0.0055, S C\left(I_{3}\right)=0.0089$, and $S C\left(I_{4}\right)=0.0066$. So, the IVTSFNs are ranked as:

$$
I_{1}>I_{3}>I_{4}>I_{2}
$$

And we get:

$$
\begin{aligned}
I_{\alpha(1)} & =([0.3,0.6],[0.2,0.7],[0.5,0.8]) \\
I_{\alpha(2)} & =([0.3,0.3],[0.4,0.5],[0.7,0.8]) \\
I_{\alpha(3)} & =([0.1,0.5],[0.2,0.2],[0.3,0.5]) \\
I_{\alpha(4)} & =([0.2,0.4],[0.2,0.6],[0.3,0.8])
\end{aligned}
$$

Now, the aggregated value of $I_{1}, I_{2}, I_{3}$, and $I_{4}$, using the IVTSFOWA operator, is given by:

$$
\begin{gathered}
\operatorname{IVTSFOWG}\left(I_{1}, I_{3}, I_{4}, I_{2}\right) \\
=([0.2081,0.4212],[0.2532,0.4336],[0.5844,0.7626])
\end{gathered}
$$

Next, the notion of the IVTSFHG operator is proposed.

Definition 12. The IVTSFHG operator for IVTSFNs $I_{j}(j=1,2,3 \ldots m)$ is of the form:

$$
\operatorname{IVTSFHG}\left(I_{1}, I_{2}, I_{3} \ldots I_{m}\right)=\prod_{j=1}^{m} \dot{I}_{\alpha(j)}^{w_{j}}
$$

where $\dot{I}_{j}$ can be computed as $\dot{I}_{j}=I_{j}^{m \mathfrak{w}}, \dot{I}_{\alpha(j)}$ denotes the $j^{\text {th }}$ largest value of $\dot{I}_{j}$, and $\mathfrak{w}=\left(\mathfrak{w}_{1}, \mathfrak{w}_{2}, \mathfrak{w}_{3} \ldots \mathfrak{w}_{n}\right)^{T}$ is the weight vector of $I_{j}$.

Theorem 11. The aggregated value of IVTSFNs $I_{j}$, using the IVTSFHG operator, is an IVTSFN and is given as:

$$
\begin{aligned}
& \operatorname{IVTSFHG}\left(I_{1}, I_{2}, I_{3} \ldots I_{m}\right) \\
& =\left(\left[\prod_{j=1}^{m}\left(\dot{s}_{\alpha(j)}^{l}\right)^{w_{j}}, \prod_{j=1}^{m}\left(\dot{s}_{\alpha(j)}^{u}\right)^{w_{j}}\right],\left[\prod_{j=1}^{m}\left(\dot{i}_{\alpha(j)}^{l}\right)^{w_{j}}, \prod_{j=1}^{m}\left(\dot{i}_{\alpha(j)}^{u}\right)^{w_{j}}\right],\left[\sqrt[q]{1-\prod_{j=1}^{m}\left(1-\left(\dot{d}_{\alpha(j)}^{l}\right)^{q}\right)^{w_{j}}}, \sqrt[q]{1-\prod_{j=1}^{m}\left(1-\left(\dot{d}_{\alpha(j)}^{u}\right)^{q}\right)^{w_{j}}}\right]\right)
\end{aligned}
$$

Proof. This result can analogously be proven as similar to that of the proof of Theorem 8 .

Example 5. Let $I_{1}=([0.3,0.6],[0.2,0.7],[0.5,0.8]), I_{2}=([0.2,0.4],[0.2,0.6],[0.3,0.8]), I_{3}=$ $([0.3,0.3],[0.4,0.5],[0.7,0.8])$, and $I_{4}=([0.1,0.5],[0.2,0.2],[0.3,0.5])$ be the four IVTSFNs for $q=4$ and let $\mathfrak{w}=(0.22,0.34,0.27,0.17)^{T}$ be the weight vector of IVTSFNs. Furthermore, let $w=$ $(0.2,0.1,0.3,0.4)^{T}$ be the aggregated associated weight vector. Then, using $\dot{I}_{j}=I_{j}^{m \mathfrak{w}}$, we have:

$$
\begin{aligned}
& \dot{I}_{1}=I_{1}^{m \mathfrak{w}} \\
& =\left(\left[(0.3)^{4 \times 0.22},(0.6)^{4 \times 0.22}\right],\left[(0.2)^{4 \times 0.22},(0.7)^{4 \times 0.22}\right],\left[\sqrt[4]{1-\left(1-(0.5)^{4}\right)^{4 \times 0.22}}, \sqrt[4]{1-\left(1-(0.8)^{4}\right)^{4 \times 0.22}}\right]\right) \\
& =([0.0018,0.0285],[0.0004,0.0528],[0.5434,0.8217])
\end{aligned}
$$

Similarly,

$$
\begin{aligned}
& \dot{I}_{2}=I_{2}^{m \mathfrak{w}}=([0.0005,0.0087],[0.0005,0.0441],[0.1945,0.7382]) \\
& \dot{I}_{3}=I_{3}^{m \mathfrak{w}}=([0.0022,0.0022],[0.0069,0.0069],[0.4730,0.7858]) \\
& \dot{I}_{4}=I_{4}^{m \mathfrak{w}}=([0.0003,0.0044],[0.0003,0.0014],[0.4410,0.8592])
\end{aligned}
$$


Now, using the score function defined in Definition 6, we have $S C\left(\dot{I}_{1}\right)=0.0020, S C\left(\dot{I}_{2}\right)=0.0011$, $S C\left(\dot{I}_{3}\right)=0.0013$, and $S C\left(\dot{I}_{4}\right)=0.0004$. So, the IVTSFNs $\dot{I}_{j}$ are ranked as:

$$
\dot{I}_{1}>\dot{I}_{3}>\dot{I}_{2}>\dot{I}_{4}
$$

Now, the aggregated value of $\dot{I}_{1}, \dot{I}_{2}, \dot{I}_{3}$, and $\dot{I}_{4}$, using the IVTSFHG operator, is given by:

$$
\operatorname{IVTSFHG}\left(I_{1}, I_{3}, I_{2}, I_{4}\right)=([0.0008,0.0055],[0.0008,0.0066],[0.4681,0.8235])
$$

Theorem 12. If $w=\left(\frac{1}{m}, \frac{1}{m}, \frac{1}{m}, \ldots \frac{1}{m}\right)^{T}$, then the IVTSFHG operator becomes the IVTSFWG operator.

Proof. As $\dot{I}_{j}=I_{j}^{m \mathfrak{w}_{j}}$ and $w=\left(\frac{1}{m}, \frac{1}{m}, \frac{1}{m}, \ldots \frac{1}{m}\right)^{T}$. So $\dot{I}_{j}^{w_{j}}=I_{j}^{\mathfrak{w}_{j}}$ and

$$
\operatorname{IVTSFHG}\left(I_{1}, I_{2}, I_{3} \ldots I_{m}\right)=\prod_{j=1}^{m} \dot{I}_{\alpha(j)}^{w_{j}}=\prod_{j=1}^{m} I_{j}^{\mathfrak{w}_{j}}=\operatorname{IVTSFWG}\left(I_{1}, I_{2}, I_{3} \ldots I_{m}\right)
$$

\section{Multi-Attribute Decision-Making Investment Planning}

MADM is one of the most suitable areas where the aggregation of the tools of fuzzy structures is applied to get useful results. A detailed literature survey of MADM problems has been discussed in Section 1. In this section, our aim is to develop the MADM method in the context of IVTSFSs.

In a MADM problem, the selection of the best or most suitable option is carried out using a list of options. Consider a list of $m$ alternatives, denoted by $A_{i}$, being observed by decision makers with $k$ attributes, denoted by $H_{k}$. The decision makers evaluated the $m$ number of alternatives with $k$ attributes and provided their information in the form of IVTSFNs. Furthermore, $w=\left(w_{1}, w_{2}, w_{3}, \ldots w_{k}\right)^{T}$ denoted the weight vector of the attributes. The detailed steps of the MADM process are given as:

Step 1: The decision makers assess the alternatives, given the attributes, and provide their information in the form of a decision matrix.

Step 2: Apply the proposed aggregation tools to the decision matrix obtained in Step 1.

Step 3: Compute the scores of the IVTSFNs obtained in Step 2.

Step 4: Analyze the score values of the alternatives and rank them to obtain the best alternative.

The following example demonstrates the proposed MADM algorithm in detail.

Example 6. A multinational company needed to announce its policy on its investment for the upcoming financial year. Keeping in mind its previous performance, the company needed to evaluate its financial policies to launch the best investment. It had four policies to be evaluated after some initial screening. These four policies included four possible places (countries) for investments i.e., $A_{1}$ : To invest in Pakistan, $A_{2}$ : To invest in Iran, $A_{3}$; To invest in the UAE, and $A_{4}$ : To invest in Bangladesh. Assuming that all of the attributes are of benefit, the four investment plans were evaluated based on the following attributes: $H_{1}$ : Comfort zone, $H_{2}$ : Government regulations, $\mathrm{H}_{3}$ : Interest of the people, and $\mathrm{H}_{4}$ : Competition in the markets. The attribute $\mathrm{H}_{1}$ shows that evaluation policies are based on the law and considers the situation of the country for the investment. The attribute $\mathrm{H}_{2}$ allows the decision makers to evaluate the policy, keeping in mind the relevant government policies that directly affect the business policies. The attribute $\mathrm{H}_{3}$ allows the decision makers to evaluate the policy based on the cultural values and overall lifestyle of the people of the country, as any investment policy is directly linked to the consumers and distributors. The fourth attribute, $H_{4}$, provides a chance for the decision makers to evaluate the policy by considering the competition in the market. If the competition in the market is low, then the 
success of the policy may be assured. All of the four factors had a great impact on the success of the policy and the decision makers provided their evaluations with all four attributes in mind. Let $w=(0.22,0.34,0.27,0.17)^{T}$ be the weight vector of the attributes. The policy makers gave their opinions in the form of IVTSFNs, as follows.

The description of imprecise information, in terms of intervals instead of crisp numbers, provides consistent and improved results numerically, as described in Section 3. Furthermore, the description of imprecision using the four membership grades, i.e., membership, abstinence, non-membership, and the refusal degree, improves the accuracy of the human opinion about an uncertain event. Therefore, the evaluation of the investment policy, in this case, is studied in the environment of IVTSFSs, using the WA and WG aggregation operators. The stepwise demonstration of the MADM process is as follows: Step 1: The evaluation of alternatives, $A_{i}$, by the decision makers in the form of decision matrix $D_{i j}$.

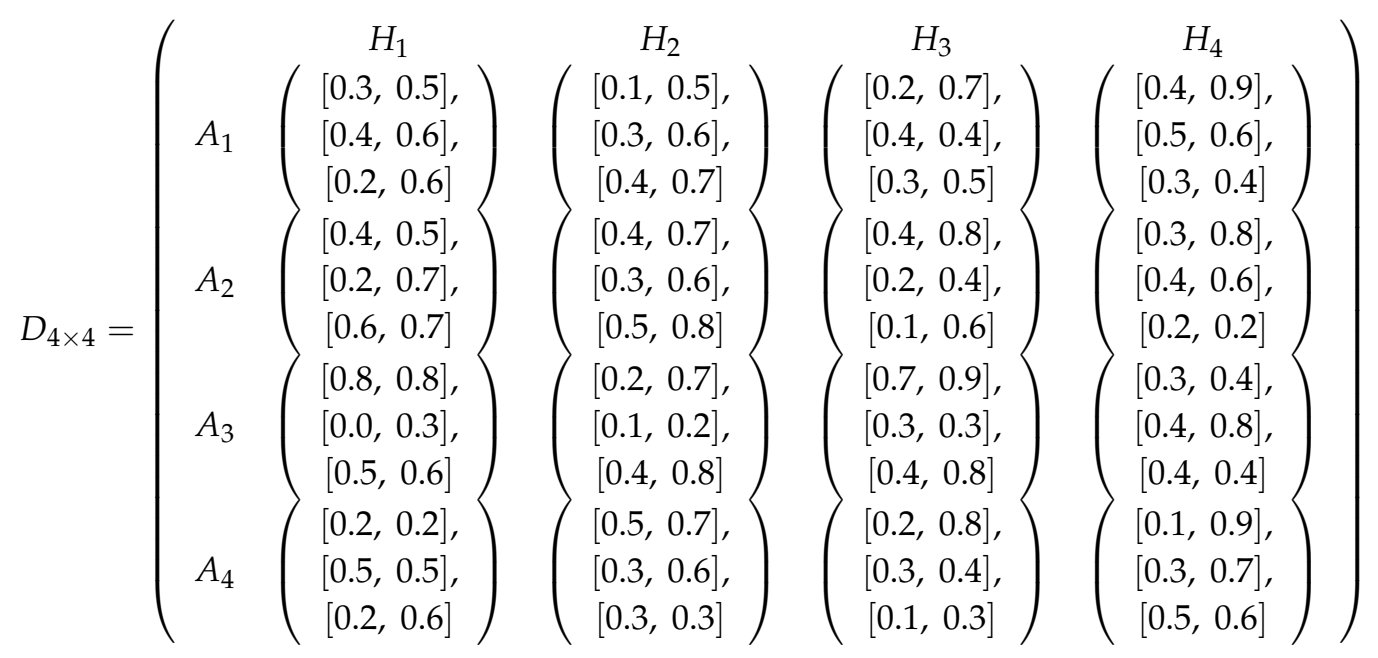

The selection of the best location for investment is carried out using two approaches. Firstly, by using the IVTSFWA operators and secondly, by using IVTSFWG operators. It can be seen that all of the values in the decision matrix are IVTSFNs for $q=5$. For a lesser $q$, some values are not IVTSFNs. Therefore, we take $q=5$.

Step 2: By applying the IVTSFWA operators to the decision matrix provided in Step 1, we get:

$$
\begin{gathered}
A_{1}=\operatorname{IVTSFWA}\left(A_{11}, A_{12}, A_{13}, A_{14}\right) \\
=([0.2986,0.7225],[0.3768,0.5378],[0.3026,0.5618]) \\
=([0.3891,0.7365],[0.2583,0.5563],[0.2884,0.5679]) \\
A_{2}=\operatorname{IVTSFWA}\left(A_{21}, A_{22}, A_{23}, A_{24}\right) \\
=([0.6634,0.7986],[0.0000,0.3088],[0.4201,0.6675]) \\
A_{3}=\operatorname{IVTSFWA}\left(A_{31}, A_{32}, A_{33}, A_{34}\right) \\
=([0.4050,0.7725],[0.3357,0.5304],[0.2225,0.3931])
\end{gathered}
$$

Step 3: This step involves the computation of the score values as such:

$$
\operatorname{SC}\left(A_{1}\right)=0.1083, \operatorname{SC}\left(A_{2}\right)=0.1423, \operatorname{SC}\left(A_{3}\right)=0.3846, \text { and } S C\left(A_{4}\right)=0.1846
$$

Step 4: In this step, the score values obtained in Step 3 are analyzed. Based on the score values, the ranking of the alternatives is given:

$$
S C\left(A_{3}\right)>S C\left(A_{4}\right)>S C\left(A_{2}\right)>S C\left(A_{1}\right)
$$


The ranking analysis shows that $A_{3}$ has the greatest value, hence the policy of making investments in the UAE is the best, according to the proposed decision-making method using the IVTSFWA operators.

Now, the same data is analyzed to find the best policy of investment using IVTSFWG operators. Step 1: We use the same evaluation of alternatives, $A_{i}$, in the form of decision matrix $D_{i j}$ as above. Step 2: By applying the IVTSFWG operators to the decision matrix provided in Step 1, we get:

$$
\begin{gathered}
A_{1}=\operatorname{IVTSFWG}\left(A_{11}, A_{12}, A_{13}, A_{14}\right) \\
=([0.1944,0.6051],[0.3768,0.5378],[0.3413,0.6132]) \\
=([0.3809,0.6894],[0.2583,0.5563],[0.4900,0.7090]) \\
A_{2}=\operatorname{IVTSFWG}\left(A_{21}, A_{22}, A_{23}, A_{24}\right) \\
=([0.4077,0.7015],[0.0000,0.3088],[0.4312,0.7455]) \\
A_{3}=\operatorname{IVTSFWG}\left(A_{31}, A_{32}, A_{33}, A_{34}\right) \\
=([0.2427,0.5749],[0.3357,0.5304],[0.3627,0.5040])
\end{gathered}
$$

Step 3: This step involves the computation of the score values as:

$$
S C\left(A_{1}\right)=0.0520, C\left(A_{2}\right)=0.0993, S C\left(A_{3}\right)=0.1263, \text { and } S C\left(A_{4}\right)=0.0589
$$

Step 4: In this step, the score values obtained in Step 3 are analyzed. Based on the score values, the ranking of alternatives is given as:

$$
S C\left(A_{3}\right)>S C\left(A_{2}\right)>S C\left(A_{4}\right)>S C\left(A_{1}\right)
$$

The ranking analysis shows that $A_{3}$ has the greatest value, thus the policy of making investments in the UAE is the best, according to the proposed decision-making method using IVTSFWG operators. Hence, in this case, we obtain the same results by using IVTAFWA operators as we do by using IVTSFWG operators. However, we will not necessarily always obtain the same results using different aggregation tools, i.e., the results obtained using IVTSFWA and IVTSFWG operators may differ. Thus, this type of information cannot be processed by using the pre-existing aggregation tools because of their limited structures. Our proposed aggregation operators, on the other hand, can aggregate such types of information. The other interesting fact is that the information provided in this problem is similar to human opinion, as it describes the degree membership, abstinence, non-membership, and the refusal degree. Furthermore, if we used only a single crisp value for each membership grade instead of intervals, then the result would drastically change, as illustrated in Section 3.

\section{Consequences of the Proposed Work and a Comparative Study}

The aim of this section is to analyze some consequences of our proposed work. It can be seen that, in some fuzzy algebraic structures, work on the aggregation tools is yet to be initiated. Therefore, some weighted averaging and weighted geometric aggregation tools for other fuzzy algebraic structures will be proposed. If the aggregation tools of a fuzzy framework already exist, then those aggregation tools will be compared with the new proposed aggregation tools.

Consider the IVTSFWA and IVTSFWG operators, as in Equations (1) and (4) respectively.

$$
\begin{aligned}
& \operatorname{IVTSFWA}\left(I_{1}, I_{2}, I_{3} \ldots I_{m}\right) \\
& =\left(\left[\sqrt[q]{1-\prod_{j=1}^{m}\left(1-\left(s_{j}^{l}\right)^{q}\right)^{w_{j}}}, \sqrt[q]{1-\prod_{j=1}^{m}\left(1-\left(s_{j}^{u}\right)^{q}\right)^{w_{j}}}\right],\left[\prod_{j=1}^{m}\left(i_{j}^{l}\right)^{w_{j}}, \prod_{j=1}^{m}\left(i_{j}^{u}\right)^{w_{j}}\right],\left[\prod_{j=1}^{m}\left(d_{j}^{l}\right)^{w_{j}}, \prod_{j=1}^{m}\left(d_{j}^{u}\right)^{w_{j}}\right]\right)
\end{aligned}
$$




$$
\begin{aligned}
& \operatorname{IVTSFWG}\left(I_{1}, I_{2}, I_{3} \ldots I_{m}\right) \\
& =\left(\left[\prod_{j=1}^{m}\left(s_{j}^{l}\right)^{w_{j}}, \prod_{j=1}^{m}\left(s_{j}^{u}\right)^{w_{j}}\right],\left[\prod_{j=1}^{m}\left(i_{j}^{l}\right)^{w_{j}}, \prod_{j=1}^{m}\left(i_{j}^{u}\right)^{w_{j}}\right],\left[\sqrt[q]{1-\prod_{j=1}^{m}\left(1-\left(d_{j}^{l}\right)^{q}\right)^{w_{j}}}, \sqrt[q]{1-\prod_{j=1}^{m}\left(1-\left(d_{j}^{u}\right)^{q}\right)^{w_{j}}}\right]\right)
\end{aligned}
$$

(1) If we use $s^{l}=s^{u}=s, i^{l}=i^{u}=i$, and $d^{l}=d^{u}=d$, then the weighted averaging and the weighted geometric aggregation operators of TSFSs are obtained and are given as:

$$
\begin{gathered}
\operatorname{TSFWA}\left(I_{1}, I_{2}, I_{3} \ldots I_{m}\right)=\left(\sqrt[q]{1-\prod_{j=1}^{m}\left(1-\left(s_{j}\right)^{q}\right)^{w_{j}}}, \prod_{j=1}^{m}\left(i_{j}\right)^{w_{j}}, \prod_{j=1}^{m}\left(d_{j}\right)^{w_{j}}\right) \\
\operatorname{TSFWG}\left(I_{1}, I_{2}, I_{3} \ldots I_{m}\right)\left(\prod_{j=1}^{m}\left(s_{j}\right)^{w_{j}}, \prod_{j=1}^{m}\left(i_{j}\right)^{w_{j}}, \sqrt[q]{1-\prod_{j=1}^{m}\left(1-\left(d_{j}\right)^{q}\right)^{w_{j}}}\right)
\end{gathered}
$$

(2) If we use $q=2$, then the weighted averaging and the weighted geometric aggregation operators of IVSFSs are obtained and are given as:

$$
\begin{aligned}
& \begin{array}{l}
\operatorname{IVSFWA}\left(I_{1}, I_{2}, I_{3} \ldots I_{m}\right) \\
=\left(\left[\sqrt{1-\prod_{j=1}^{m}\left(1-\left(s_{j}^{l}\right)^{2}\right)^{w_{j}}}, \sqrt{1-\prod_{j=1}^{m}\left(1-\left(s_{j}^{u}\right)^{2}\right)^{w_{j}}}\right],\left[\prod_{j=1}^{m}\left(i_{j}^{l}\right)^{w_{j}}, \prod_{j=1}^{m}\left(i_{j}^{u}\right)^{w_{j}}\right],\left[\prod_{j=1}^{m}\left(d_{j}^{l}\right)^{w_{j}}, \prod_{j=1}^{m}\left(d_{j}^{u}\right)^{w_{j}}\right]\right)
\end{array} \\
& \operatorname{IVSFWG}\left(I_{1}, I_{2}, I_{3} \ldots I_{m}\right) \\
& =\left(\left[\prod_{j=1}^{m}\left(s_{j}^{l}\right)^{w_{j}}, \prod_{j=1}^{m}\left(s_{j}^{u}\right)^{w_{j}}\right],\left[\prod_{j=1}^{m}\left(i_{j}^{l}\right)^{w_{j}}, \prod_{j=1}^{m}\left(i_{j}^{u}\right)^{w_{j}}\right],\left[\sqrt{1-\prod_{j=1}^{m}\left(1-\left(d_{j}^{l}\right)^{2}\right)^{w_{j}}}, \sqrt{1-\prod_{j=1}^{m}\left(1-\left(d_{j}^{u}\right)^{2}\right)^{w_{j}}}\right]\right)
\end{aligned}
$$

(1) If we use $q=2, s^{l}=s^{u}=s, i^{l}=i^{u}=i$, and $d^{l}=d^{u}=d$, then the weighted averaging and the weighted geometric aggregation operators of SFSs are obtained and are given as:

$$
\begin{gathered}
\operatorname{SFWA}\left(I_{1}, I_{2}, I_{3} \ldots I_{m}\right)=\left(\sqrt{\left.1-\prod_{j=1}^{m}\left(1-\left(s_{j}\right)^{2}\right)^{w_{j}}, \prod_{j=1}^{m}\left(i_{j}\right)^{w_{j}}, \prod_{j=1}^{m}\left(d_{j}\right)^{w_{j}}\right)}\right. \\
\operatorname{SFWG}\left(I_{1}, I_{2}, I_{3} \ldots I_{m}\right)\left(\prod_{j=1}^{m}\left(s_{j}\right)^{w_{j}}, \prod_{j=1}^{m}\left(i_{j}\right)^{w_{j}}, \sqrt{1-\prod_{j=1}^{m}\left(1-\left(d_{j}\right)^{2}\right)^{w_{j}}}\right)
\end{gathered}
$$

(2) If we use $q=1$, then the weighted averaging and the weighted geometric aggregation operators of IVPFSs are obtained and are given as:

$$
\begin{aligned}
& \operatorname{IVPFWA}\left(I_{1}, I_{2}, I_{3} \ldots I_{m}\right) \\
& =\left(\left[1-\prod_{j=1}^{m}\left(1-\left(s_{j}^{l}\right)\right)^{w_{j}}, 1-\prod_{j=1}^{m}\left(1-\left(s_{j}^{u}\right)\right)^{w_{j}}\right],\left[\prod_{j=1}^{m}\left(i_{j}^{l}\right)^{w_{j}}, \prod_{j=1}^{m}\left(i_{j}^{u}\right)^{w_{j}}\right],\left[\prod_{j=1}^{m}\left(d_{j}^{l}\right)^{w_{j}}, \prod_{j=1}^{m}\left(d_{j}^{u}\right)^{w_{j}}\right]\right) \\
& \operatorname{IVPFWG}\left(I_{1}, I_{2}, I_{3} \ldots I_{m}\right) \\
& =\left(\left[\prod_{j=1}^{m}\left(s_{j}^{l}\right)^{w_{j}}, \prod_{j=1}^{m}\left(s_{j}^{u}\right)^{w_{j}}\right],\left[\prod_{j=1}^{m}\left(i_{j}^{l}\right)^{w_{j}}, \prod_{j=1}^{m}\left(i_{j}^{u}\right)^{w_{j}}\right],\left[1-\prod_{j=1}^{m}\left(1-\left(d_{j}^{l}\right)^{2}\right)^{w_{j}}, 1-\prod_{j=1}^{m}\left(1-\left(d_{j}^{u}\right)^{2}\right)^{w_{j}}\right]\right)
\end{aligned}
$$

(3) If we use $q=1, s^{l}=s^{u}=s, i^{l}=i^{u}=i$, and $d^{l}=d^{u}=d$, then the weighted averaging and the weighted geometric aggregation operators of PFSs are obtained and are given as:

$$
\operatorname{PFWA}\left(I_{1}, I_{2}, I_{3} \ldots I_{m}\right)=\left(1-\prod_{j=1}^{m}\left(1-\left(s_{j}\right)\right)^{w_{j}}, \prod_{j=1}^{m}\left(i_{j}\right)^{w_{j}}, \prod_{j=1}^{m}\left(d_{j}\right)^{w_{j}}\right)
$$




$$
\operatorname{PFWG}\left(I_{1}, I_{2}, I_{3} \ldots I_{m}\right)\left(\prod_{j=1}^{m}\left(s_{j}\right)^{w_{j}}, \prod_{j=1}^{m}\left(i_{j}\right)^{w_{j}}, 1-\prod_{j=1}^{m}\left(1-\left(d_{j}\right)\right)^{w_{j}}\right)
$$

Note that this PFWA operator is the same as the one proposed by Garg [50].

(4) If we use $i^{l}=i^{u}=i=0$, then the weighted averaging and the weighted geometric aggregation operators of the newly developed IVq-ROPFSs are obtained and are given as:

$$
\begin{aligned}
& \operatorname{IVQROPFWA}\left(I_{1}, I_{2}, I_{3} \ldots I_{m}\right) \\
& =\left(\left[\sqrt[q]{1-\prod_{j=1}^{m}\left(1-\left(s_{j}^{l}\right)^{q}\right)^{w_{j}}}, \sqrt[q]{1-\prod_{j=1}^{m}\left(1-\left(s_{j}^{u}\right)^{q}\right)^{w_{j}}}\right],\left[\prod_{j=1}^{m}\left(d_{j}^{l}\right)^{w_{j}}, \prod_{j=1}^{m}\left(d_{j}^{u}\right)^{w_{j}}\right]\right) \\
& \operatorname{IVQROPFWG}\left(I_{1}, I_{2}, I_{3} \ldots I_{m}\right) \\
& =\left(\left[\prod_{j=1}^{m}\left(s_{j}^{l}\right)^{w_{j}}, \prod_{j=1}^{m}\left(s_{j}^{u}\right)^{w_{j}}\right],\left[\sqrt[q]{1-\prod_{j=1}^{m}\left(1-\left(d_{j}^{l}\right)^{q}\right)^{w_{j}}}, \sqrt[q]{1-\prod_{j=1}^{m}\left(1-\left(d_{j}^{u}\right)^{q}\right)^{w_{j}}}\right]\right)
\end{aligned}
$$

(5) If we use $s^{l}=s^{u}=s, i^{l}=i^{u}=i=0$, and $d^{l}=d^{u}=d$, then the weighted averaging and the weighted geometric aggregation operators of the newly developed q-ROPFSs are obtained and are given as:

$$
\begin{gathered}
\operatorname{QROPFWA}\left(I_{1}, I_{2}, I_{3} \ldots I_{m}\right)=\left(\sqrt[q]{1-\prod_{j=1}^{m}\left(1-\left(s_{j}\right)^{q}\right)^{w_{j}}}, \prod_{j=1}^{m}\left(d_{j}\right)^{w_{j}}\right) \\
\operatorname{QROPFWG}\left(I_{1}, I_{2}, I_{3} \ldots I_{m}\right)\left(\prod_{j=1}^{m}\left(s_{j}\right)^{w_{j}}, \sqrt[q]{1-\prod_{j=1}^{m}\left(1-\left(d_{j}\right)^{q}\right)^{w_{j}}}\right)
\end{gathered}
$$

(6) If we use $q=2$ and $i^{l}=i^{u}=i=0$, then the weighted averaging and the weighted geometric aggregation operators of the IVPyFSs are obtained and are given as:

$$
\begin{aligned}
& \operatorname{IVPyFWA}\left(I_{1}, I_{2}, I_{3} \ldots I_{m}\right) \\
& =\left(\left[\sqrt{1-\prod_{j=1}^{m}\left(1-\left(s_{j}^{l}\right)^{2}\right)^{w_{j}}}, \sqrt{1-\prod_{j=1}^{m}\left(1-\left(s_{j}^{u}\right)^{2}\right)^{w_{j}}}\right],\left[\prod_{j=1}^{m}\left(d_{j}^{l}\right)^{w_{j}}, \prod_{j=1}^{m}\left(d_{j}^{u}\right)^{w_{j}}\right]\right) \\
& \operatorname{IVPyFWG}\left(I_{1}, I_{2}, I_{3} \ldots I_{m}\right) \\
& =\left(\left[\prod_{j=1}^{m}\left(s_{j}^{l}\right)^{w_{j}}, \prod_{j=1}^{m}\left(s_{j}^{u}\right)^{w_{j}}\right],\left[\sqrt{1-\prod_{j=1}^{m}\left(1-\left(d_{j}^{l}\right)^{2}\right)^{w_{j}}}, \sqrt{1-\prod_{j=1}^{m}\left(1-\left(d_{j}^{u}\right)^{2}\right)^{w_{j}}}\right]\right)
\end{aligned}
$$

Note that these IVPyFWA and IWPyFWG operators are the same as those proposed by Rehman et al. [41,42].

(7) If we use $q=2, s^{l}=s^{u}=s, i^{l}=i^{u}=i=0$, and $d^{l}=d^{u}=d$, then the weighted averaging and the weighted geometric aggregation operators of the PyFSs are obtained and are given as:

$$
\begin{gathered}
\operatorname{PyFWA}\left(I_{1}, I_{2}, I_{3} \ldots I_{m}\right)=\left(\sqrt{1-\prod_{j=1}^{m}\left(1-\left(s_{j}\right)^{2}\right)^{w_{j}}}, \prod_{j=1}^{m}\left(d_{j}\right)^{w_{j}}\right) \\
\operatorname{PyFWG}\left(I_{1}, I_{2}, I_{3} \ldots I_{m}\right)\left(\prod_{j=1}^{m}\left(s_{j}\right)^{w_{j}}, \sqrt{1-\prod_{j=1}^{m}\left(1-\left(d_{j}\right)^{2}\right)^{w_{j}}}\right)
\end{gathered}
$$

Note that these PyFWA and PyFWG operators are the same as those proposed by Rahman et al. and Peng and Yang [39,40]. 
(8) If we use $q=1$ and $i^{l}=i^{u}=i=0$, then the weighted averaging and the weighted geometric aggregation operators of the IVIFSs are obtained and are given as:

$$
\begin{aligned}
& \operatorname{IVIFWA}\left(I_{1}, I_{2}, I_{3} \ldots I_{m}\right) \\
& =\left(\left[1-\prod_{j=1}^{m}\left(1-\left(s_{j}^{l}\right)\right)^{w_{j}}, 1-\prod_{j=1}^{m}\left(1-\left(s_{j}^{u}\right)\right)^{w_{j}}\right],\left[\prod_{j=1}^{m}\left(i_{j}^{l}\right)^{w_{j}}, \prod_{j=1}^{m}\left(i_{j}^{u}\right)^{w_{j}}\right],\left[\prod_{j=1}^{m}\left(d_{j}^{l}\right)^{w_{j}}, \prod_{j=1}^{m}\left(d_{j}^{u}\right)^{w_{j}}\right]\right) \\
& \operatorname{IVIFWG}\left(I_{1}, I_{2}, I_{3} \ldots I_{m}\right) \\
& =\left(\left[\prod_{j=1}^{m}\left(s_{j}^{l}\right)^{w_{j}}, \prod_{j=1}^{m}\left(s_{j}^{u}\right)^{w_{j}}\right],\left[\prod_{j=1}^{m}\left(i_{j}^{l}\right)^{w_{j}}, \prod_{j=1}^{m}\left(i_{j}^{u}\right)^{w_{j}}\right],\left[1-\prod_{j=1}^{m}\left(1-\left(d_{j}^{l}\right)^{2}\right)^{w_{j}}, 1-\prod_{j=1}^{m}\left(1-\left(d_{j}^{u}\right)^{2}\right)^{w_{j}}\right]\right)
\end{aligned}
$$

Note that these IVIFWA and IVIFWG operators are the same as those proposed by Wang et al. and Wei and Wang $[29,30]$.

(9) If we use $q=1, s^{l}=s^{u}=s, i^{l}=i^{u}=i=0$, and $d^{l}=d^{u}=d$, then the weighted averaging and the weighted geometric aggregation operators of the PFSs are obtained and are given as:

$$
\begin{gathered}
\operatorname{IFWA}\left(I_{1}, I_{2}, I_{3} \ldots I_{m}\right)=\left(1-\prod_{j=1}^{m}\left(1-\left(s_{j}\right)\right)^{w_{j}}, \prod_{j=1}^{m}\left(d_{j}\right)^{w_{j}}\right) \\
\operatorname{IFWG}\left(I_{1}, I_{2}, I_{3} \ldots I_{m}\right)\left(\prod_{j=1}^{m}\left(s_{j}\right)^{w_{j}}, 1-\prod_{j=1}^{m}\left(1-\left(d_{j}\right)\right)^{w_{j}}\right)
\end{gathered}
$$

Note that these IFWA and IFWG operators are the same as those proposed by Xu and Yager $[24,25]$.

Hence, using the proposed definitions of the IVTSFWA and IVTSFWG operators and with the help of some restrictions, we successfully proposed weighted averaging and weighted geometric aggregation operators for the IVq-ROPFSs, q-ROPFSs, TSFSs, IVSFSs, SFSs, IVPFSs, and PFSs. Furthermore, it is also shown that the proposed operators are generalizations of the aggregation tools of PFSs, PyFSs, IVPyFSs, IFSs, and IVIFs.

Now, we discuss the viability of the proposed IVTSF aggregation operators in the problems of the existing frameworks.

Firstly, consider the MADM problem discussed by Mahmood et al. [22], with three alternatives and three attributes. The decision matrix is shown in Table 3.

Table 3. The decision matrix with alternatives $A_{i}$ and attributes $H_{j}$.

\begin{tabular}{cccc}
\hline & $\boldsymbol{H}_{1}$ & $\boldsymbol{H}_{2}$ & $\boldsymbol{H}_{3}$ \\
\hline$A_{1}$ & $(0.8,0.5,0.4)$ & $(0.7,0.4,0.4)$ & $(0.3,0.5,0.4)$ \\
$A_{2}$ & $(0.9,0.2,0.4)$ & $(0.6,0.3,0.2)$ & $(0.4,0.1,0.7)$ \\
$A_{3}$ & $(0.5,0.5,0.5)$ & $(0.8,0.2,0.3)$ & $(0.6,0.4,0.3)$ \\
\hline
\end{tabular}

This type of MADM problem can easily be solved by placing $s^{l}=s^{u}=s, i^{l}=i^{u}=i$ and $d^{l}=d^{u}=d$ into the proposed IVTSFWA and IVTSFWG operators, i.e., by applying Equation (7) and Equation (8).

Now, consider the MADM problem studied by Garg [51], with four alternatives and four attributes. The decision matrix is shown in Table 4.

Table 4. The decision matrix with alternatives $A_{i}$ and attributes $H_{j}$.

\begin{tabular}{ccccc}
\hline & $\boldsymbol{H}_{1}$ & $\boldsymbol{H}_{2}$ & $\boldsymbol{H}_{3}$ & $\boldsymbol{H}_{3}$ \\
\hline$A_{1}$ & $(0.2,0.1,0.6)$ & $(0.5,0.3,0.1)$ & $(0.5,0.1,0.3)$ & $(0.4,0.3,0.2)$ \\
$A_{2}$ & $(0.1,0.4,0.4)$ & $(0.6,0.3,0.1)$ & $(0.5,0.2,0.2)$ & $(0.2,0.1,0.7)$ \\
$A_{3}$ & $(0.3,0.2,0.2)$ & $(0.6,0.2,0.1)$ & $(0.4,0.1,0.3)$ & $(0.3,0.3,0.4)$ \\
$A_{4}$ & $(0.3,0.1,0.6)$ & $(0.1,0.2,0.6)$ & $(0.1,0.3,0.5)$ & $(0.2,0.3,0.2)$ \\
\hline
\end{tabular}


This type of MADM problem can easily be solved by placing $q=1, s^{l}=s^{u}=s, i^{l}=i^{u}=i$, and $d^{l}=d^{u}=d$ into the proposed IVTSFWA operator, i.e., by using Equation (15).

Similarly, the MADM problem solved by Wang et al. [52] can also be solved using Equations (15) and (16).

\section{Advantages}

In this section, the advantages of the proposed new aggregation tools over the pre-existing aggregation tools are discussed and further demonstrated with a numerical example.

If we consider Example 6 of Section 7, it becomes quite clear that none of the existing aggregation tools used in Equations (9) to (30) of the fuzzy algebraic frameworks could solve the data involved in that problem, thus showing the limitations of the existing operators.

On the other hand, if we consider an example in the context of the existing fuzzy structure, then such a problem can easily be solved by using the proposed aggregation tools of the IVTSFSs (by using Equations (7) and (8)). For instance, consider a decision matrix of four alternatives and three attributes in the MADM discussed by Garg [10].

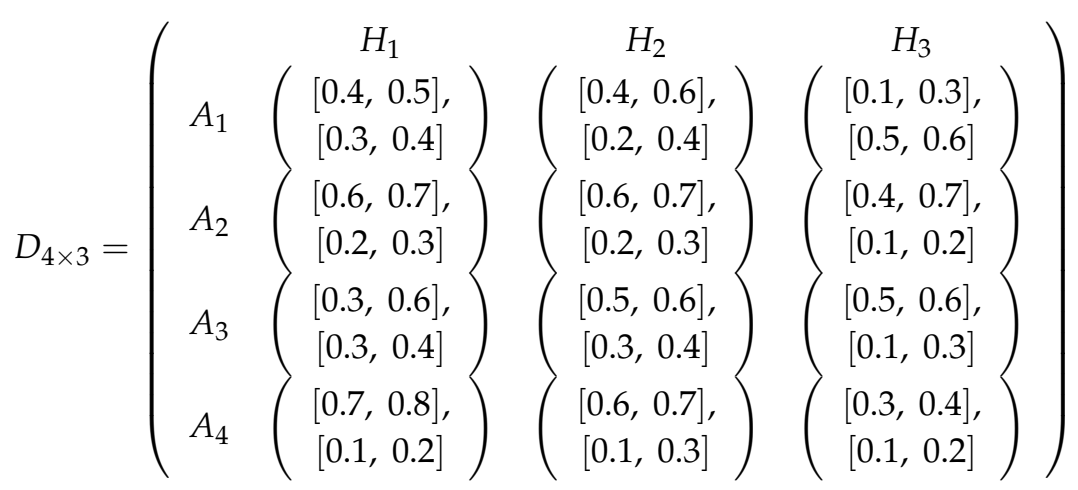

Here, the four alternatives denote four possible policies of investment and our aim is to find the best policy. Now, this problem can easily be solved using the aggregation operators of the IVTSFNs (using Equations (7) and (8)) by including $q=2$ and $i^{l}=i^{u}=i=0$. The IVTSFWA operator for $q=2$ and $i^{l}=i^{u}=i=0$, as discussed in Equation (23), becomes:

$$
\begin{aligned}
& \operatorname{IVPyFWA}\left(I_{1}, I_{2}, I_{3} \ldots I_{m}\right) \\
& =\left(\left[\sqrt{1-\prod_{j=1}^{m}\left(1-\left(s_{j}^{l}\right)^{2}\right)^{w_{j}}}, \sqrt{1-\prod_{j=1}^{m}\left(1-\left(s_{j}^{u}\right)^{2}\right)^{w_{j}}}\right],\left[\prod_{j=1}^{m}\left(d_{j}^{l}\right)^{w_{j}}, \prod_{j=1}^{m}\left(d_{j}^{u}\right)^{w_{j}}\right]\right)
\end{aligned}
$$

The aggregation results are:

$$
\begin{aligned}
& A_{1}=\operatorname{IVTSFWA}\left(A_{11}, A_{12}, A_{13}\right)=([0.2692,0.4079],[0.3955,0.5206]) \\
& A_{2}=\operatorname{IVTSFWA}\left(A_{11}, A_{12}, A_{13}\right)=([0.4586,0.6408],[0.1995,0.3153]) \\
& A_{3}=\operatorname{IVTSFWA}\left(A_{11}, A_{12}, A_{13}\right)=([0.3972,0.5612],[0.2197,0.3946]) \\
& A_{4}=\operatorname{IVTSFWA}\left(A_{11}, A_{12}, A_{13}\right)=([0.4910,0.6024],[0.1565,0.2736])
\end{aligned}
$$

Using the score function, we get:

$$
\operatorname{SC}\left(A_{2}\right)>S C\left(A_{4}\right)>S C\left(A_{3}\right)>S C\left(A_{1}\right)
$$

Hence, the score values show that $A_{2}$ is the best policy to be implemented. This result is well in accordance with the results obtained by Garg [10]. Therefore, the claim that the IVTSFWA and IVTSFWG operators can deal with data in the form of pre-existing fuzzy structures is proven to be true. 


\section{Conclusions}

The concept of the IVTSFS is defined and its novelty and significance are demonstrated. The basic operations of IVTSFSs are developed and, with the help of some restrictions, it is shown that the proposed new operations are more applicable than the existing ones. The theory of aggregation operators for IVTSFSs have also been developed as some weighted averaging and weighted geometric aggregation operators have been introduced. The validity of the averaging and geometric aggregation operators was analyzed using the induction method and the characteristics of the monotonicity, boundedness, and idempotency were studied. Some ordered weighted averaging (geometric) and hybrid averaging (geometric) aggregation operators were also developed, keeping in mind the ranking of importance. Adhering to some restrictions over the proposed work, aggregation operators for IVq-ROPFSs, q-ROPFSs, and IVPFSs were also developed. The MADM method in the context of the IVTSFS was established and a numerical example was solved using both IVTSFWA and IVTSFWG operators to understand the proposed MADM method. The advantages of the new proposed work are discussed with the help of examples. It is clearly explained that the concept of the IVTSF can process the information which is sufficiently close to human nature, but it can also handle the information provided in the context of IFSs, PFSs, PyFSs, q-ROPFs, IVIFSs, IVPyFSs, and IVq-ROPFS. On the other hand, none of the pre-existing operators could process such information. In the future, we aim to develop some similarity, as well as distance, measures for IVTSFSs and study their usefulness in pattern recognition, MADM, and clustering. Furthermore, the McLaurin symmetric mean operator and the Bonferroni mean operator can also be developed for IVTSFSs. We also aim to develop the idea of Einstein and interactive operators for IVTSFSs and to study their viability in MADM for future research.

Author Contributions: Conceptualization, K.U. and T.M.; Data curation, K.U. and N.J.; Formal analysis, T.M. and N.H.; Funding acquisition, M.H.; Investigation, K.U. and N.H.; Methodology, K.U., T.M., and N.H.; Project administration, T.M., M.H., and N.H.; Software, K.U. and N.J.; Supervision, T.M. and N.H.; Validation, K.U., T.M., N.H., and N.J.; Writing—original draft, K.U., T.M., and N.J.; Writing—review and editing, K.U., T.M., and N.H.

Funding: This research was funded by Universiti Putra Malaysia under vote number 9001103.

Acknowledgments: This work was supported in part by Universiti Putra Malaysia.

Conflicts of Interest: The authors declare no conflicts of interest.

\section{References}

1. Zadeh, L.A. Fuzzy sets. Inf. Control 1965, 8, 338-353. [CrossRef]

2. Gorzalczany, M.B. A method of inference in approximate reasoning based on interval-valued fuzzy sets. Fuzzy Sets Syst. 1987, 21, 1-17. [CrossRef]

3. Atanassov, K. Intuitionistic fuzzy sets. Fuzzy Sets Syst. 1986, 20, 87-96. [CrossRef]

4. Atanassov, K.; Gargov, K. Interval valued intuitionistic fuzzy sets. Fuzzy Sets Syst. 1989, 31, $343-349$. [CrossRef]

5. Yager, R.R. Pythagorean fuzzy subsets. In Proceedings of the 2013 Joint IFSA World Congress and NAFIPS Annual Meeting (IFSA/NAFIPS), Edmonton, AB, Canada, 24-28 June 2013. [CrossRef]

6. Yager, R.R. Generalized orthopair fuzzy sets. IEEE Trans. Fuzzy Syst. 2017, 25, 1222-1230. [CrossRef]

7. Peng, X.; Yang, Y. Fundamental properties of interval-valued Pythagorean fuzzy aggregation operators. Int. J. Intell. Syst. 2016, 31, 444-487. [CrossRef]

8. Joshi, B.P.; Singh, A.; Bhatt, P.K.; Vaisla, K.S. Interval valued q-rung orthopair fuzzy sets and their properties. J. Intell. Fuzzy Syst. 2018, 35, 5225-5230. [CrossRef]

9. Garg, H. A new generalized improved score function of interval-valued intuitionistic fuzzy sets and applications in expert systems. Appl. Soft Comput. 2016, 38, 988-999. [CrossRef]

10. Garg, H. A novel accuracy function under interval-valued Pythagorean fuzzy environment for solving multicriteria decision making problem. J. Intell. Fuzzy Syst 2016, 31, 529-540. [CrossRef] 
11. Garg, H.; Rani, M.; Sharma, S.; Vishwakarma, Y. Intuitionistic fuzzy optimization technique for solving multi-objective reliability optimization problems in interval environment. Expert Syst. Appl. 2014, 41, 3157-3167. [CrossRef]

12. Kumar, K.; Garg, H. TOPSIS method based on the connection number of set pair analysis under interval-valued intuitionistic fuzzy set environment. Comput. Appl. Math. 2018, 37, 1319-1329. [CrossRef]

13. Garg, H.; Agarwal, N.; Tripathi, A. Entropy based multi-criteria decision making method under fuzzy environment and unknown attribute weights. Glob. J. Technol. Optim. 2015, 6, 13-20.

14. Garg, H. Linguistic Pythagorean fuzzy sets and its applications in multiattribute decision-making process. Int. J. Intell. Syst. 2018, 33, 1234-1263. [CrossRef]

15. Luo, M.; Liang, J. A Novel Similarity measure for interval-valued intuitionistic fuzzy sets and its applications. Symmetry 2018, 10, 441. [CrossRef]

16. Ye, D.; Liang, D.; Hu, P. Three-way decisions with interval-valued intuitionistic fuzzy decision-theoretic rough sets in group decision-making. Symmetry 2018, 10, 281. [CrossRef]

17. Cuong, B.C. Picture fuzzy sets. J. Comput. Sci. Cybern. 2014, 30, 409-420.

18. Wei, G. Picture fuzzy cross-entropy for multiple attribute decision making problem. J. Bus. Econ. Manag. 2016, 17, 491-502. [CrossRef]

19. Wei, G. Some cosine similarity measures for picture fuzzy sets and their applications to strategic decision making. Informatica 2017, 28, 547-564. [CrossRef]

20. Wei, G. Some similarity measures for picture fuzzy sets and their applications. Iran. J. Fuzzy Syst. 2018, 15, 77-89.

21. Wei, G.; Gao, H. The generalized dice similarity measures for picture fuzzy sets and their applications. Informatica 2018, 29, 1-18. [CrossRef]

22. Mahmood, T.; Ullah, K.; Khan, Q.; Jan, N. An approach towards decision making and medical diagnosis problems using the concept of spherical fuzzy sets. Neural Comput. Appl. 2018. [CrossRef]

23. Ullah, K.; Mahmood, T.; Jan, N. Similarity measures for T-spherical fuzzy sets with applications in pattern recognition. Symmetry 2018, 10, 193. [CrossRef]

24. Xu, Z. Intuitionistic fuzzy aggregation operators. IEEE Trans. Fuzzy Syst. 2007, 15, 1179-1187.

25. Xu, Z.; Yager, R.R. Some geometric aggregation operators based on intuitionistic fuzzy sets. Int. J. Gen. Syst. 2006, 35, 417-433. [CrossRef]

26. Zhao, H.; Xu, Z.; Ni, M.; Liu, S. Generalized aggregation operators for intuitionistic fuzzy sets. Int. J. Intell. Syst. 2010, 25, 1-30. [CrossRef]

27. Wei, G. Some induced geometric aggregation operators with intuitionistic fuzzy information and their application to group decision making. Appl. Soft Comput. 2010, 10, 423-431. [CrossRef]

28. Xu, Y.; Wang, H. The induced generalized aggregation operators for intuitionistic fuzzy sets and their application in group decision making. Appl. Soft Comput. 2012, 12, 1168-1179. [CrossRef]

29. Wang, W.; Liu, X.; Qin, Y. Interval-valued intuitionistic fuzzy aggregation operators. J. Syst. Eng. Electron. 2012, 23, 574-580. [CrossRef]

30. Wei, G.; Wang, X. Some geometric aggregation operators based on interval-valued intuitionistic fuzzy sets and their application to group decision making. In Proceedings of the 2007 International Conference on Computational Intelligence and Security (CIS 2007), Harbin, China, 15-19 December 2007. [CrossRef]

31. Yu, D. Decision making based on generalized geometric operator under interval-valued intuitionistic fuzzy environment. J. Intell. Fuzzy Syst. 2013, 25, 471-480.

32. Xu, Z.; Cai, X. Interval-valued intuitionistic fuzzy information aggregation. In Intuitionistic Fuzzy Information Aggregation; Springer: Berlin, Germany, 2012; Chapter 2; pp. 103-149.

33. Garg, H. Generalized intuitionistic fuzzy interactive geometric interaction operators using Einstein t-norm and t-conorm and their application to decision making. Comput. Ind. Eng. 2016, 101, 53-69. [CrossRef]

34. Garg, H. Some series of intuitionistic fuzzy interactive averaging aggregation operator. SpringerPlus 2016, 5, 999. [CrossRef] [PubMed]

35. Garg, H. Generalized intuitionistic fuzzy multiplicative interactive geometric operators and their application to multiple criteria decision making. Int. J. Mach. Learn. Cybern. 2016, 7, 1075-1092. [CrossRef]

36. Garg, H.; Kumar, K. Some aggregation operators for linguistic intuitionistic fuzzy set and its application to group decision-making process using the set pair analysis. Arab. J. Sci. Eng. 2018, 43, 3213-3227. [CrossRef] 
37. Garg, H.; Arora, R. Bonferroni mean aggregation operators under intuitionistic fuzzy soft set environment and their applications to decision-making. J. Oper. Res. Soc. 2018, 69, 1711-1724. [CrossRef]

38. Joshi, D.; Kumar, S. Improved accuracy function for interval-valued intuitionistic fuzzy sets and its application to multi-attributes group decision making. Cybern. Syst. 2018, 49, 64-76. [CrossRef]

39. Rahman, K.; Abdullah, S.; Husain, F.; Khan, M.S.A. Approaches to Pythagorean fuzzy geometric aggregation operators. Int. J. Comput. Sci. Inf. Secur. 2016, 14, 174-200.

40. Peng, X.; Yang, Y. Some results for Pythagorean fuzzy sets. Int. J. Intell. Syst. 2015, 30, 1133-1160. [CrossRef]

41. Rahman, K.; Abdullah, S.; Shakeel, M.; Khan, M.S.A.; Ullah, M. Interval-valued Pythagorean fuzzy geometric aggregation operators and their application to group decision making problem. Cogent Math. Stat. 2017, 4, 1338638. [CrossRef]

42. Rahman, K.; Ali, A.; Khan, M.S.A. Some interval-valued Pythagorean fuzzy weighted averaging aggregation operators and their application to multiple attribute decision making. Punjab. Univ. J. Math. 2018, 50, 113-129.

43. Zhu, J.; Li, Y. Pythagorean fuzzy Muirhead mean operators and their application in multiple-criteria group decision-Making. Information 2018, 9, 142. [CrossRef]

44. Peng, X.; Yuan, H. Fundamental properties of Pythagorean fuzzy aggregation operators. Fundam. Inform. 2016, 147, 415-446. [CrossRef]

45. Ma, Z.; Xu, Z. Symmetric Pythagorean fuzzy weighted geometric/averaging operators and their application in multicriteria decision-making problems. Int. J. Intell. Syst. 2016, 31, 1198-1219. [CrossRef]

46. Garg, H. Hesitant Pythagorean fuzzy sets and their aggregation operators in multiple attribute decision-making. Int. J. Uncertain. Quantif. 2018, 8, 167-189. [CrossRef]

47. Garg, H. Generalised Pythagorean fuzzy geometric interactive aggregation operators using Einstein operations and their application to decision making. J. Exp. Theor. Artif. Intell. 2018, 30, 763-794. [CrossRef]

48. Ullah, K.; Mahmood, T.; Jan, N.; Broumi, S.; Khan, Q. On bipolar-valued hesitant fuzzy sets and their applications in multi-attribute decision making. Nucleus 2018, 55, 85-93.

49. Mahmood, T.; Ullah, K.; Jan, N.; Deli, I.; Khan, Q. Some aggregation operators for bipolar-valued hesitant fuzzy information based on einstein operational laws. J. Eng. Appl. Sci. 2017, 36, 63-72.

50. Mahmood, T.; Ullah, K.; Khan, Q.; Smarandache, F. Some aggregation operators for bipolar-valued hesitant fuzzy information. J. Fundam. Appl. Sci. 2017, 10, 240-250.

51. Garg, H. Some picture fuzzy aggregation operators and their applications to multicriteria decision-making. Arab. J. Sci. Eng. 2017, 42, 5275-5290. [CrossRef]

52. Wang, C.; Zhou, X.; Tu, H.; Tao, S. Some geometric aggregation operators based on picture fuzzy sets and their application in multiple attribute decision making. Ital. J. Pure Appl. Math. 2017, 37, 477-492.

53. Ullah, K.; Mahmood, T.; Jan, N. Some averaging aggregation operators for t-spherical fuzzy sets and their applications in multi-attribute decision making. In Proceedings of the International Conference on Soft Computing \& Machine Learning (ICSCML), Wuhan, China, 26-28 April 2019.

54. Garg, H.; Munir, M.; Ullah, K.; Mahmood, T.; Jan, N. Algorithm for T-spherical fuzzy multi-attribute decision making based on improved interactive aggregation operators. Symmetry 2018, 10, 670. [CrossRef]

55. Abdel-Basset, M.; Manogaran, G.; Gamal, A.; Smarandache, F. A group decision making framework based on neutrosophic TOPSIS approach for smart medical device selection. J. Med. Syst. 2019, 43, 38. [CrossRef] [PubMed]

56. Abdel-Basset, M.; Gunasekaran, M.; Mohamed, M.; Chilamkurti, N. A framework for risk assessment, management and evaluation: Economic tool for quantifying risks in supply chain. Future Gener. Comput. Syst. 2019, 90, 489-502. [CrossRef]

57. Basset, M.A.; Mohamed, M.; Sangaiah, A.K.; Jain, V. An integrated neutrosophic AHP and SWOT method for strategic planning methodology selection. Benchmarking 2018, 25, 2546-2564. [CrossRef]

58. Abdel-Basset, M.; Mohamed, M.; Hussien, A.N.; Sangaiah, A.K. A novel group decision-making model based on triangular neutrosophic numbers. Soft Comput. 2018, 22, 6629-6643. [CrossRef]

59. Abdel-Basset, M.; Mohamed, M.; Smarandache, F. A hybrid neutrosophic group ANP-TOPSIS framework for supplier selection problems. Symmetry 2018, 10, 226. [CrossRef]

60. Abdel-Basset, M.; Zhou, Y.; Mohamed, M.; Chang, V.A. group decision making framework based on neutrosophic VIKOR approach for e-government website evaluation. J. Intell. Fuzzy Syst. 2018, 34, 4213-4224. [CrossRef] 
61. Abdel-Basset, M.; Manogaran, G.; Gamal, A.; Smarandache, F. A hybrid approach of neutrosophic sets and DEMATEL method for developing supplier selection criteria. Des. Autom. Embed. Syst. 2018, 22, 257-278. [CrossRef]

62. Chang, V.; Abdel-Basset, M.; Ramachandran, M. Towards a reuse strategic decision pattern framework-from theories to practices. Inf. Syst. Front. 2018, 1-18. [CrossRef]

63. Abdel-Basset, M.; Mohamed, M.; Smarandache, F.; Chang, V. Neutrosophic association rule mining algorithm for big data analysis. Symmetry 2018, 10, 106. [CrossRef]

64. Al-Qudah, Y.; Hassan, N. Complex multi-fuzzy soft set: Its entropy and similarity measure. IEEE Access 2018, 6, 65002-65017. [CrossRef]

65. Alhazaymeh, K.; Hassan, N. Vague soft set relations and functions. J. Intell. Fuzzy Syst. 2015, 28, 1205-1212.

66. Al-Qudah, Y.; Hassan, N. Bipolar fuzzy soft expert set and its application in decision making. Int. J. Appl. Decis. Sci. 2017, 10, 175-191. [CrossRef]

67. Adam, F.; Hassan, N. Q-fuzzy soft matrix and its application. AIP Conf. Proc. 2014, 1602, 772-778.

68. Adam, F.; Hassan, N. Properties on the multi Q-fuzzy soft matrix. AIP Conf. Proc. 2014, 1614, 834-839.

69. Al-Quran, A.; Hassan, N. Neutrosophic vague soft expert set theory. J. Intell. Fuzzy Syst. 2016, 30, 3691-3702. [CrossRef]

70. Adam, F.; Hassan, N. Operations on Q-fuzzy soft set. Appl. Math. Sci. 2014, 8, 8697-8701. [CrossRef]

71. Adam, F.; Hassan, N. Q-fuzzy soft set. Appl. Math. Sci. 2014, 8, 8689-8695. [CrossRef]

72. Alhazaymeh, K.; Hassan, N. Mapping on generalized vague soft expert set. Int. J. Pure Appl. Math. 2014, 93, 369-376. [CrossRef]

73. Jan, N.; Ullah, K.; Mahmood, T.; Garg, H.; Davvaz, B.; Saeid, A.B.; Broumi, S. Some root level modifications in interval valued fuzzy graphs and their generalizations including neutrosophic graphs. Mathematics 2019, 7, 72. [CrossRef]

(C) 2019 by the authors. Licensee MDPI, Basel, Switzerland. This article is an open access article distributed under the terms and conditions of the Creative Commons Attribution (CC BY) license (http:/ / creativecommons.org/licenses/by/4.0/). 